\title{
Schanuel's theorem for heights defined via extension fields
}

\author{
CHRISTOPHER FREI AND MARTIN WIDMER
}

\begin{abstract}
Let $k$ be a number field, let $\theta$ be a nonzero algebraic number, and let $H(\cdot)$ be the Weil height on the algebraic numbers. In response to a question by T. Loher and D. W. Masser, we prove an asymptotic formula for the number of $\alpha \in k$ with $H(\alpha \theta) \leq X$, and we analyze the leading constant in our asymptotic formula. In particular, we prove a sharp upper bound in terms of the classical Schanuel constant.

We also prove an asymptotic counting result for a new class of height functions defined via extension fields of $k$ with a fairly explicit error term. This provides a conceptual framework for Loher and Masser's problem and generalizations thereof.

Finally, we establish asymptotic counting results for varying $\theta$, namely, for the number of $\sqrt{p} \alpha$ of bounded height, where $\alpha \in k$ and $p$ is any rational prime inert in $k$.
\end{abstract}

Mathematics Subject Classification (2010): 11R04, (primary); 11G50, 11D45 (secondary).

\section{Introduction}

Let $k$ be a number field. A well-known result due to Schanuel [12] shows that the subset of $k^{n}$ of points with absolute multiplicative Weil height no larger than $X$ has cardinality

$$
S_{k}(n) X^{d(n+1)}+O\left(X^{d(n+1)-1} \log X\right),
$$

as $X$ tends to infinity. Here $d$ is the degree of $k$ and the positive constant $S_{k}(n)$ involves all the classical number field invariants; for the definition see (1.2).

In the present article we generalize this result in various respects motivated by a question of Loher and Masser. Let $\theta$ be a nonzero algebraic number, let $H(\cdot)$

The first author was supported in part by the FWF project \#S9611-N23 and by a Humboldt Research Fellowship for Postdoctoral Researchers.

The second author was supported in part by the FWF grant \#M1222-N13.

Received January 17, 2014; accepted in revised form May 5, 2014.

Published online February 2016. 
denote the absolute multiplicative Weil height on the algebraic numbers $\overline{\mathbb{Q}}$, and write $N(\theta k, X)$ for the number of $\alpha \in k$ with $H(\theta \alpha) \leq X$.

Evertse was the first one to consider the quantity $N(\theta k, X)$. The proof of his celebrated uniform upper bounds [4] for the number of solutions of $S$-unit equations over $k$ involves the following uniform upper bound

$$
N(\theta k, X) \leq 5 \cdot 2^{d} X^{3 d}+1
$$

Later Schmidt [13, Lemma 8B, page 29] refined Evertse's argument to get the correct exponent on $X$. Schmidt used a different height but elementary inequalities between them imply

$$
N(\theta k, X) \leq 36 \cdot 2^{3 d} X^{2 d}
$$

But the constant is fairly large. Indeed, the constant's exponential dependence on $d$ can be removed, as shown by Loher and Masser. More precisely, they proved

$$
N(\theta k, X) \leq 68(d \log d) X^{2 d},
$$

provided $d>1$, and $N(\theta \mathbb{Q}, X) \leq 17 X^{2}$. (In the special case $\theta \in k$ a similar result was obtained earlier by Loher in his Ph.D. thesis [8].) By counting roots of unity they also showed that an upper bound with a constant of the form $o(d \log \log d)$ cannot hold, and hence regarding the degree their result is nearly optimal. Loher and Masser's result (1.1) played also an important role in the recent proof of a longstanding conjecture of Erdo"s on the largest prime divisor of $2^{n}-1$ by Stewart [16]. Stewart's strategy builds up on work of Yu [21,22] on $p$-adic logarithm forms in which Yu applies a consequence of (1.1) to obtain a significant improvement. It is this improvement that makes Stewart's approach work ( $c f$. [22, page 378]).

All the proofs of these upper bounds for $N(\theta k, X)$ rely in an essential way on the box-principle, which works well for upper bounds but seems inappropriate to produce asymptotic results. This may have motivated Loher and Masser's following statement [6, page 279] regarding their bound on $N(\theta k, X)$ : It would be interesting to know if there are asymptotic formulae like Schanuel's for the cardinalities here, at least for fixed $\theta$ not in $k$.

Our Theorem 1.1 responds to this problem for fixed $\theta$ not in $k$, and our Theorem 1.4 generalizes Theorem 1.1 to arbitrary dimensions. Theorem 1.2 gives a sharp upper bound for the leading constant in these asymptotics in terms of Schanuel's constant $S_{k}(n)$. In Theorem 1.3, we shall see asymptotic results for varying $\theta$ not in $k$.

To provide a more general framework for Loher and Masser's, and similar questions, we introduce a new class of heights on $\mathbb{P}^{n}(k)$, using finite extensions of the base field $k$. As usual, these heights decompose into local factors, one for each place $v$ of $k$. However, at a finite number of non-Archimedean places, the local factors of these heights do not necessarily arise from norms, and moreover, their values do not necessarily lie in the value groups of the corresponding places 
$v$. Theorem 6.1 (in Section 6), from which we will deduce Theorem 1.4 (and thus also Theorem 1.1), is a counting result, in the style of Schanuel's, for these heights.

Our heights are special cases of the heights used by Peyre [11, Définition 1.2]. Peyre gives asymptotic counting results [11, Corollaire 6.2.18] but no error estimates for his general heights. Therefore the main terms in our Theorem 1.4 and Theorem 6.1 could likely be derived from Peyre's result, although with a different representation of the constant. Indeed, a significant part of this work consists of finding the right representation which enables us to prove the sharp upper bound in Theorem 1.2, as well as some invariance properties. Furthermore, Peyre's approach does not seem to provide comparable error terms, and the latter are essential for the proof of our Theorem 1.3. A very recent result due to Ange [1, Théorème 1.1] provides a Schanuel type counting result for another special case of Peyre's heights. Ange also gives a completely explicit and fairly sharp error term. However, his heights require Euclidean/Hermitian norms at the Archimedean places and thus do not include the usual Weil height.

Next we introduce some notation. We start with Schanuel's constant $S_{k}(n)$, which is defined as follows

$$
S_{k}(n)=\frac{h_{k} R_{k}}{w_{k} \zeta_{k}(n+1)}\left(\frac{2^{r}(2 \pi)^{s}}{\sqrt{\left|\Delta_{k}\right|}}\right)^{n+1}(n+1)^{r+s-1} .
$$

Here $h_{k}$ is the class number, $R_{k}$ the regulator, $w_{k}$ the number of roots of unity in $k, \zeta_{k}$ the Dedekind zeta-function of $k, \Delta_{k}$ the discriminant, $r=r_{k}$ is the number of real embeddings of $k$ and $s=s_{k}$ is the number of pairs of complex conjugate embeddings of $k$.

For each place $v$ of $k$ (or $w$ of $K:=k(\theta)$ ) we choose the unique absolute value $|\cdot|_{v}$ on $k$ (or $|\cdot|_{w}$ on $K$ ) that extends either the usual Euclidean absolute value on $\mathbb{Q}$ or a usual $p$-adic absolute value. We also fix a completion $k_{v}$ of $k$ at $v$ and for each Archimedean place $v$ of $k$ we define a set of points $\left(z_{0}, \ldots, z_{n}\right) \in k_{v}^{n+1}$ by

$$
\prod_{w \mid v} \max \left\{\left|z_{0}\right|_{v},|\theta|_{w}\left|z_{1}\right|_{v}, \ldots,|\theta|_{w}\left|z_{n}\right|_{v}\right\}^{\frac{\left[K w: k k_{v}\right]}{\lfloor K: k]}}<1,
$$

where the product runs over all places $w$ of $K=k(\theta)$ extending $v$. As these sets are open, bounded, and not empty, they are measurable and have a finite, positive volume, which we denote by $V_{v}$. Here we identify $k_{v}$ with $\mathbb{R}$ or with $\mathbb{C}$, and we identify the latter with $\mathbb{R}^{2}$. We define

$$
V=V(\theta, k, n):=\left(2^{r} \pi^{s}\right)^{-(n+1)} \prod_{v \mid \infty} V_{v} .
$$

Write $\mathcal{O}_{k}$ for the ring of integers of $k$ and let $\mu_{k}$ be the Möbius function for nonzero ideals of $\mathcal{O}_{k}$. For ideals $A, B$ of $\mathcal{O}_{k}$, we write $(A, B):=A+B$. Moreover, $\mathfrak{N}_{k} A$ denotes the absolute norm of the fractional ideal $A$ of $k$. For $\alpha \in k$, we also write $\mathfrak{N}_{k}(\alpha):=\mathfrak{N}_{k}\left(\alpha \mathcal{O}_{k}\right)$. Analogous notation is used for $K$ instead of $k$. 
For an ideal $B$ of $\mathcal{O}_{k}$, we write ${ }^{\mathfrak{u}} B:=B \mathcal{O}_{K}$ for the extension of $B$ to $\mathcal{O}_{K}$ ("up"). Similarly, for an ideal $\mathfrak{D}$ of $\mathcal{O}_{K}$, we write ${ }^{\mathfrak{D}} \mathfrak{D}:=\mathfrak{D} \cap \mathcal{O}_{k}$ for the contraction of $\mathfrak{D}$ to $\mathcal{O}_{k}$ ("down").

The dependence on $\theta$ comes in two flavors; while $V$ amounts only to the Archimedean part the following constant captures both parts.

Let $\alpha$ be nonzero and in $\mathcal{O}_{k}$ such that $\alpha \theta \in \mathcal{O}_{K}$, let $\mathfrak{D}:=\alpha \theta \mathcal{O}_{K}$, and $D:=$ $\mathfrak{d} \mathfrak{D}$. We define

$$
g_{k}(\theta, n):=\frac{V}{\mathfrak{N}_{k}(\alpha)^{n}} \sum_{B \mid D} \frac{\mathfrak{N}_{K}\left(\mathfrak{D},{ }^{\mathfrak{u}} B\right)^{\frac{n+1}{[K: k]}}}{\mathfrak{N}_{k} B} \sum_{A \mid B^{-1} D} \frac{\mu_{k}(A)}{\mathfrak{N}_{k} A} \prod_{P \mid A B} \frac{\mathfrak{N}_{k} P^{n+1}-\mathfrak{N}_{k} P}{\mathfrak{N}_{k} P^{n+1}-1} .
$$

In the product, $P$ runs over all prime ideals of $\mathcal{O}_{k}$ dividing $A B$. It will follow from Lemma 2.3 that this definition does not depend on the choice of $\alpha$, and from Proposition 2.2 that $g_{k}(\theta, n)>0$.

Theorem 1.1. Let $\theta$ be a nonzero algebraic number, let $k$ be a number field and denote its degree by $d$. Then, as $X \geq 1$ tends to infinity, we have

$$
N(\theta k, X)=g_{k}(\theta, 1) S_{k}(1) X^{2 d}+O\left(X^{2 d-1} \mathfrak{L}\right),
$$

where $\mathfrak{L}:=\log (X+1)$ if $d=1$ and $\mathfrak{L}:=1$ otherwise. The implicit constant in the $O$-term depends on $\theta$ and on $k$.

Let us briefly discuss some properties of the constant $g_{k}(\theta, 1)$ and then illustrate the theorem by some examples.

For any nonzero $\alpha$ in $k$ we have $\theta k=\alpha \theta k$. Also, the height is invariant under multiplication by a root of unity. Therefore $N(\theta k, X)=N(\zeta \alpha \theta k, X)$ for any $\alpha \in k^{*}$ and any root of unity $\zeta$, in particular we have

$$
g_{k}(\theta, 1)=g_{k}(\zeta \alpha \theta, 1) .
$$

This can also be proved directly from the definition as we shall see in Section 2. By Schanuel's theorem we conclude that $g_{k}(\zeta \alpha, 1)=1$. But, as is straightforward to check, the theorem implies even $g_{k}(\zeta \alpha, 1)=1$ for $\zeta$ a root of any unit in $\mathcal{O}_{k}$ and $\alpha \in k^{*}$.

The fact that $H(\alpha \theta)=H\left(\alpha^{-1} \theta^{-1}\right)$ implies

$$
g_{k}(\theta, 1)=g_{k}\left(\theta^{-1}, 1\right) .
$$

Next we consider the problem of uniformly bounding $g_{k}(\theta, 1)$. From Schanuel's theorem and the standard inequalities $H(\alpha) / H(\theta) \leq H(\theta \alpha) \leq H(\theta) H(\alpha)$ we conclude

$$
H(\theta)^{-2 d} \leq g_{k}(\theta, 1) \leq H(\theta)^{2 d} .
$$


This raises the question of the existence of bounds that are uniform in $\theta$ or in $d$, or even uniform in both quantities $\theta$ and $d$. From (1.1) we obtain an upper bound that is uniform in $\theta$, i.e., for $d>1$

$$
g_{k}(\theta, 1) \leq \frac{68 d \log d}{S_{k}(1)} .
$$

Now if we fix $d>1$ and vary the fields $k$ then by the Siegel-Brauer theorem the right hand-side tends to infinity, so this bound really depends on $\Delta_{k}$ and not only on $d$. However, intuitively one might guess that for most $\alpha \in k$ one has $H(\theta \alpha) \geq H(\alpha)$, so one might even expect that $g_{k}(\theta, 1) \leq 1$ holds true, which, of course, would be best-possible. We shall answer here all of these questions. We start with the upper bound and confirm the intuitive guess.

Theorem 1.2. Let $\theta$ be a nonzero algebraic number. Then $g_{k}(\theta, n) \leq 1$. Moreover, equality holds if and only if for every place $v$ of $k$ there is an $\alpha_{v} \in k_{v}$ such that $|\theta|_{w}=\left|\alpha_{v}\right|_{v}$ holds for all places $w$ of $K$ above $v$.

Let us now illustrate Theorem 1.1 with an example, and thereby explain also the situation regarding lower bounds for $g_{k}(\theta, 1)$. Let us first take $k=\mathbb{Q}$, and $\theta=\sqrt{p}$ for a prime number $p$. Then we get the asymptotics

$$
\frac{2 \sqrt{p}}{p+1} S_{\mathbb{Q}}(1) X^{2}=\frac{24 \sqrt{p}}{\pi^{2}(p+1)} X^{2} .
$$

More generally, if $p$ is inert in $k$ and $\theta=\sqrt{p}$ then we get the asymptotics

$$
\frac{2 p^{d / 2}}{p^{d}+1} S_{k}(1) X^{2 d}
$$

Letting $p$ tend to infinity shows that there is no lower bound for $g_{k}(\theta, 1)$ that is uniform in $\theta$. Likewise, fixing a $p$ and taking a sequence $\mathbb{Q}, k_{1}, k_{2}, \ldots$ of number fields with $p$ inert in $k_{i}$ and $\left[k_{i}: \mathbb{Q}\right] \rightarrow \infty$ shows that there is no lower bound for $g_{k}(\theta, 1)$ that is uniform in $d$.

The fast decay of $g_{k}(\sqrt{p}, 1)$ as $p$ runs over the set $\mathbf{P}_{k}$ (which we define as the set of positive rational primes inert in $k$ ) suggests another problem. Let

$$
\sqrt{\mathbf{P}_{k}} k:=\left\{\sqrt{p} \alpha: p \in \mathbf{P}_{k}, \alpha \in k\right\}=\bigcup_{p \in \mathbf{P}_{k}} \sqrt{p} k .
$$

The above set has uniformly bounded degree, and thus, by Northcott's theorem, we may consider its counting function $N\left(\sqrt{\mathbf{P}_{k}} k, X\right):=\left|\left\{\beta \in \sqrt{\mathbf{P}_{k}} k: H(\beta) \leq X\right\}\right|$. Now if $d>2$ then the sum over the terms in (1.6) converges, so it is natural to ask whether the asymptotics of $N\left(\sqrt{\mathbf{P}_{k}} k, X\right)$ are given simply by summing the asymptotics of $N(\sqrt{p} k, X)$ over $\mathbf{P}_{k}$. The following result positively answers this question. 
Theorem 1.3. Let $k$ be a number field of degree $d$. Then, as $X \geq 3$ tends to infinity, we have

$$
N\left(\sqrt{\mathbf{P}_{k}} k, X\right)= \begin{cases}S_{k}(1) X^{4} \log \log X+O\left(X^{4}\right) & \text { if } d=2, \\ \left(\sum_{\mathbf{P}_{k}} \frac{2 p^{d / 2}}{p^{d}+1}\right) S_{k}(1) X^{2 d}+O\left(X^{2 d-1} \mathcal{L}\right) & \text { if } d>2,\end{cases}
$$

where $\mathcal{L}=\log \log X$ if $d=3$ and $\mathcal{L}=1$ if $d>3$. The implicit constant in the $O$-term depends on $k$.

The case $d=2$ is just slightly more difficult than $d>2$ and requires additionally Chebotarev's density theorem and partial summation. However, it is not clear to us how to handle the case $d=1$.

Finally, let us mention that Theorem 1.1 can also be used to count the elements in the nonzero, e.g., square classes $k^{*} /\left(k^{*}\right)^{2}$. Each class has the form $\gamma \cdot\left(k^{*}\right)^{2}$ with some $\gamma \in k^{*}$. To count the number $N\left(\gamma \cdot\left(k^{*}\right)^{2}, X\right)$ of elements in this square class with height no larger than $X$ we note that $H\left(\gamma \alpha^{2}\right)=H(\sqrt{\gamma} \alpha)^{2}$, and thus $N\left(\gamma \cdot\left(k^{*}\right)^{2}, X\right)=(1 / 2)(N(\sqrt{\gamma} k, \sqrt{X})-1) . E . g$. , the square class $\left(\mathbb{Q}^{*}\right)^{2}$ has asymptotically $\left(6 / \pi^{2}\right) X$ elements whereas the square class $11 \cdot\left(\mathbb{Q}^{*}\right)^{2}$ has asymptotically only $\left(\sqrt{11} / \pi^{2}\right) X$ elements of height bounded by $X$.

Next we generalize Theorem 1.1 to higher dimensions. Let $N\left(\theta k^{n}, X\right)$ be the number of points $\boldsymbol{\alpha}=\left(\alpha_{1}, \ldots, \alpha_{n}\right) \in k^{n}$ with $H\left(\left(\theta \alpha_{1}, \ldots, \theta \alpha_{n}\right)\right) \leq X$. Of course, here $H: \overline{\mathbb{Q}}^{n} \rightarrow[1, \infty)$ is the (affine) absolute multiplicative Weil height, defined by

$$
H\left(\omega_{1}, \ldots, \omega_{n}\right)^{[K: \mathbb{Q}]}:=\prod_{w \in M_{K}} \max \left\{1,\left|\omega_{1}\right|_{w}, \ldots,\left|\omega_{n}\right|_{w}\right\}^{d_{w}},
$$

where $K$ is any number field containing $\omega_{1}, \ldots, \omega_{n}$, the index $w$ runs over the set $M_{K}$ of all places of $K$, and $d_{w}:=\left[K_{w}: \mathbb{Q}_{w}\right]$ denotes the local degree, where $\mathbb{Q}_{w}$ is the completion of $\mathbb{Q}$ with respect to the place below $w$.

Theorem 1.4. Let $\theta$ be a nonzero algebraic number, let $k$ be a number field, denote its degree by $d$, and let $n$ be a positive rational integer. Then, as $X \geq 1$ tends to infinity, we have

$$
N\left(\theta k^{n}, X\right)=g_{k}(\theta, n) S_{k}(n) X^{d(n+1)}+O\left(X^{d(n+1)-1} \mathfrak{L}\right),
$$

where $\mathfrak{L}:=\log (X+1)$ if $(n, d)=(1,1)$, and $\mathfrak{L}:=1$ otherwise. The implicit constant in the $O$-term depends on $\theta$, on $k$, and on $n$.

Of course the invariance property (1.5) remains valid for arbitrary $n$ instead of 1. Ange [1, Corollaire 1.6] has shown a related result (although with different choice of the height); instead of fixing one $\theta$ he allows a different $\theta$ for each coordinate and his error term is completely explicit and quite sharp. On the other hand he requires that a (positive) power of each $\theta$ lies in the ground field $k$. 
So far we have counted elements $\theta \boldsymbol{\alpha}$ in $\theta k^{n}$ of bounded height. What if we replace the set $\theta k$ by $\theta+k$ ? Or $\theta k^{2}$ by $\theta_{1} k \times \theta_{2} k$ ? More generally, we suppose $L_{1}, \ldots, L_{n}$ are linearly independent linear forms in $n$ variables with coefficients in $\overline{\mathbb{Q}}$ and $\theta_{1}, \ldots, \theta_{n}$ are in $\overline{\mathbb{Q}}$. Suppose we want to count elements of bounded height in the set

$$
\left\{\left(L_{1}(\boldsymbol{\alpha})+\theta_{1}, \ldots, L_{n}(\boldsymbol{\alpha})+\theta_{n}\right): \boldsymbol{\alpha} \in k^{n}\right\} .
$$

Now let $\boldsymbol{\alpha}:=\left(\omega_{1} / \omega_{0}, \ldots, \omega_{n} / \omega_{0}\right) \in k^{n}$ and define $\omega:=\left(\omega_{0}, \ldots, \omega_{n}\right)$. Then

$$
H\left(\left(L_{1}(\boldsymbol{\alpha})+\theta_{1}, \ldots, L_{n}(\boldsymbol{\alpha})+\theta_{n}\right)\right)=\prod_{w} \max \left\{\left|\mathcal{L}_{0}(\boldsymbol{\omega})\right|_{w}, \ldots,\left|\mathcal{L}_{n}(\boldsymbol{\omega})\right|_{w}\right\}^{\frac{\left[K_{w}: \mathbb{Q} w\right]}{[K: \mathbb{Q}]}}
$$

where $\mathcal{L}_{0}(\boldsymbol{\omega})=\omega_{0}$ and $\mathcal{L}_{i}(\boldsymbol{\omega})=L_{i}\left(\omega_{1}, \ldots, \omega_{n}\right)+\theta_{i} \omega_{0}($ for $1 \leq i \leq n)$, which give us $n+1$ linearly independent linear forms. Here the right hand-side defines a special case of a so-called adelic Lipschitz height $H_{\mathcal{N}}$ (introduced in [19]) on $\mathbb{P}^{n}(K)$, where $K$ is any number field containing $k$, and the coefficients of $\mathcal{L}_{0}, \ldots, \mathcal{L}_{n}$, and the product runs over all places $w$ of $K$. Thus, we need to count the points $P=\left(\omega_{0}: \cdots: \omega_{n}\right) \in \mathbb{P}^{n}(k)$ with $\omega_{0} \neq 0$ and $H_{\mathcal{N}}(P) \leq X$.

These generalizations of Loher and Masser's problem naturally motivate our general theorem (Theorem 6.1), which is as follows. Given two number fields $k \subseteq$ $K$ and an adelic Lipschitz height $H_{\mathcal{N}}$ on $K$, we give an asymptotic formula for the number of points $P \in \mathbb{P}^{n}(k)$ with $H_{\mathcal{N}}(P) \leq X$, as the parameter $X$ tends to infinity. To be more accurate, we also impose a minor additional assumption on the adelic Lipschitz height $H_{\mathcal{N}}$, which seems fulfilled in all natural applications, in particular, it holds in the aforementioned examples.

The special case $K=k$ of our general theorem follows from a result in [19]. There, a complementary result was proved, in the sense that points of $\mathbb{P}^{n}(K)$ defined over a proper subextension of $K / k$ were excluded from the counting (which is insignificant for the main term but was needed to obtain good error terms).

Now already with general linear forms as above it seems unlikely that the main term can be brought into an as civilized form as for Theorem 1.4 (see also the remark in [18, page 1766 third paragraph]). Indeed, a considerable part of our work consists of finding the simple representation of the constant in the special case of Theorem 1.4. However, it turns out that the given representation is not so convenient for theoretical considerations. Indeed, even the most obvious properties, such as the invariance property (1.5), are not immediately clear from the present definition. In Section 2 we establish a representation of $g_{k}(\theta, n)$ as a product of local factors (Proposition 2.2), which is a first step in the proof of Theorem 1.2 and also reveals the invariance property (1.5).

At any rate, a situation involving linear forms similar to the above turns up if we want to count solutions of a system of linear equations with certain restrictions to the coordinates of the solutions. Here is an example. Consider the equation

$$
\sqrt{2} x+\sqrt{3} y+\sqrt{5} z=0
$$


defined over $K=\mathbb{Q}(\sqrt{2}, \sqrt{3}, \sqrt{5})$. Using arguments from [18] one can easily compute that the number of solutions $(x, y, z) \in K^{3}$ with $H((x, y, z)) \leq X$ is asymptotically given by

$$
\left(\frac{\sqrt{96}-(\sqrt{2}+\sqrt{3}-\sqrt{5})^{2}}{\sqrt{480}}\right)^{8} S_{K}(2) X^{24}+O\left(X^{23}\right) .
$$

But what about the number of such solutions whose first two coordinates are rational? This question reduces to counting the elements $\left(\omega_{0}: \omega_{1}: \omega_{2}\right) \in \mathbb{P}^{2}(\mathbb{Q})$ with bounded adelic Lipschitz height

$$
H_{\mathcal{N}}\left(\left(\omega_{0}: \omega_{1}: \omega_{2}\right)\right)=\prod_{w} \max \left\{\left|\omega_{0}\right|_{w},\left|\omega_{1}\right|_{w},\left|\omega_{2}\right|_{w},\left|\frac{\sqrt{2} \omega_{1}+\sqrt{3} \omega_{2}}{\sqrt{5}}\right|_{w}\right\}^{\frac{\left[K w: \mathbb{Q}_{w}\right]}{[K: \mathbb{Q}]}} .
$$

Applying our general theorem gives the following asymptotic formula

$$
N_{L}(X)=V_{\mathcal{N}^{\prime}} \cdot \frac{1}{62 \zeta(3)} \cdot\left(1+2 \cdot 5^{1 / 4}+4 \cdot 5^{-1 / 2}\right) X^{3}+O\left(X^{2}\right)
$$

for the number $N_{L}(X)$ of solutions $(x, y, z)$ of (1.7) of height bounded by $X$ and with $x, y \in \mathbb{Q}$. Here $V_{\mathcal{N}^{\prime}}$ denotes the volume of the set of points $\left(z_{0}, z_{1}, z_{2}\right)$ in $\mathbb{R}^{3}$ that satisfy the inequality

$\max \left\{\left|z_{0}\right|,\left|z_{1}\right|,\left|z_{2}\right|,\left|\sqrt{2} z_{1}+\sqrt{3} z_{2}\right| / \sqrt{5}\right\} \max \left\{\left|z_{0}\right|,\left|z_{1}\right|,\left|z_{2}\right|,\left|\sqrt{2} z_{1}-\sqrt{3} z_{2}\right| / \sqrt{5}\right\}<1$.

For the computations we refer the reader to the appendix.

Finally, by Northcott's theorem there is no need to restrict to a fixed number field, and one could also consider all number fields of a given fixed degree simultaneously. Let us define the set

$$
\theta k(n ; e)=\left\{\left(\theta \alpha_{1}, \ldots, \theta \alpha_{n}\right):\left[k\left(\alpha_{1}, \ldots, \alpha_{n}\right): k\right]=e\right\} .
$$

So Theorem 1.4 gives the asymptotics for the counting function $N(\theta k(n ; 1), X)=$ $N\left(\theta k^{n}, X\right)$, and more generally, one could ask for the asymptotics of $N(\theta k(n ; e), X)$. The special case $\theta \in k$ was considered in [5,9,10,14,15], and [17]. Indeed, it is likely that the methods from [19] and [17], combined with those of the present article, are sufficient to solve this problem, provided $n$ is large enough. On the other hand, it would be interesting to know whether Masser and Vaaler's approach from [9] can be combined with ours to handle the case $n=1$.

The plan of the paper is as follows. In Section 2 we establish a product representation of $g_{k}(\theta, n)$, and we use this to deduce some of its properties. This product form is also the starting point in the proof of Theorem 1.2, which we give in Section 3. Then in Section 4 we state and prove some basic facts about lattice points, which are required for the proofs of Theorem 6.1 and Theorem 1.3. Section 5 provides the necessary notions such as adelic Lipschitz systems to state our general 
theorem. Then in Section 6 we state the general theorem (Theorem 6.1), and in Section 7 we give its proof. From Theorem 6.1 we deduce Theorem 1.4, which is done in Section 8. The proof of Theorem 1.3 is carried out in Section 9. Finally, in the appendix we calculate formula (1.8) using Theorem 6.1.

By a prime ideal we always mean a nonzero prime ideal. By $E \unlhd \mathcal{O}_{k}$, we mean that $E$ is a nonzero ideal of $\mathcal{O}_{k}$. An empty product is always interpreted as 1 , and an empty sum is interpreted as 0 .

ACKNOWLEDGEMENTS. We would like to thank David Masser for having brought our attention to some of the problems considered here, and Robert Tichy for giving us the opportunity to start this collaboration during our common time in Graz. This work was completed while the second author was a Visiting Fellow of the Center for Advanced Studies at LMU München in August 2012. He would like to thank Ulrich Derenthal for the invitation, and the CAS for the financial support. Finally, we are indebted to the referee for the very careful reading and an excellent report with many valuable and detailed suggestions. The referee also alerted us to an error in the statement of Lemma A.1, which is now corrected.

\section{Product representation and invariance properties of the constant}

In this section, we use a product representation for the constant $g_{k}(\theta, n)$ to derive some of its properties. Let $\mathfrak{D}, B$ be nonzero ideals of $\mathcal{O}_{K}$ or $\mathcal{O}_{k}$, respectively. For convenience, we define

$$
q(\mathfrak{D}, B):=q(\mathfrak{D}, B, n):=\frac{\mathfrak{N}_{K}\left(\mathfrak{D},{ }^{\mathfrak{u}} B\right)^{(n+1) /[K: k]}}{\mathfrak{N}_{k} B} .
$$

Clearly, $q(\mathfrak{D}, B)$ is multiplicative in $B$, by which we mean that $q\left(\mathfrak{D}, B_{1} B_{2}\right)=$ $q\left(\mathfrak{D}, B_{1}\right) q\left(\mathfrak{D}, B_{2}\right)$ whenever $\left(B_{1}, B_{2}\right)=1$. Moreover, $q(\mathfrak{D}, B)=q\left(\left(\mathfrak{D},{ }^{\mathfrak{u}} B\right), B\right)$, and if $B_{1} \mid B_{2}$, then $q\left({ }^{\mathfrak{u}} B_{2} \mathfrak{D}, B_{1}\right)=\mathfrak{N}_{k} B_{1}^{n}$ and $q\left({ }^{\mathfrak{u}} B_{1} \mathfrak{D}, B_{2}\right)=\mathfrak{N}_{k} B_{1}^{n} q\left(\mathfrak{D}, B_{1}^{-1} B_{2}\right)$.

We now define local factors at prime ideals $P$ of $\mathcal{O}_{k}$, by

$$
g_{P}(\mathfrak{D}, n):=\frac{\mathfrak{N}_{k} P-1}{\mathfrak{N}_{k} P^{n+1}-1}\left(1+\left(\mathfrak{N}_{k} P^{n}-1\right) \sum_{j=0}^{\infty} q\left(\mathfrak{D}, P^{j}\right)\right) .
$$

Let $v_{P}$ denote the $P$-adic valuation on $k$, normalized by $v_{P}\left(k^{*}\right)=\mathbb{Z}$. The infinite sum converges, since

$$
q\left(\mathfrak{D}, P^{j}\right)=\mathfrak{N}_{k} P^{v_{P}(\mathfrak{d} \mathfrak{D})-j} q\left(\mathfrak{D}, P^{v_{P}(\mathfrak{d} \mathfrak{D})}\right)
$$

holds for all $j \geq v_{P}\left({ }^{\mathfrak{d}} \mathfrak{D}\right)$. Clearly, $g_{P}(\mathfrak{D}, n)=g_{P}\left(\mathfrak{D}_{P}, n\right)$, where $\mathfrak{D}_{P}:=$ $\prod_{\mathfrak{P} \mid P} \mathfrak{P}^{v} \mathfrak{P}^{(\mathfrak{D})}$ is the part of $\mathfrak{D}$ lying over $P$. 
Lemma 2.1. Let $\mathfrak{D}$ be a nonzero ideal of $\mathcal{O}_{K}$ and $D:={ }^{\mathfrak{d}} \mathfrak{D}$. Then

$$
\sum_{B \mid D} q(\mathfrak{D}, B) \sum_{A \mid B^{-1} D} \frac{\mu_{k}(A)}{\mathfrak{N}_{k} A} \prod_{P \mid A B} \frac{\mathfrak{N}_{k} P^{n+1}-\mathfrak{N}_{k} P}{\mathfrak{N}_{k} P^{n+1}-1}=\prod_{P} g_{P}(\mathfrak{D}, n) .
$$

Proof. We start by investigating the expression

$$
S(D, B):=\sum_{A \mid B^{-1} D} \frac{\mu_{k}(A)}{\mathfrak{N}_{k} A} \prod_{P \mid A B} \frac{\mathfrak{N}_{k} P^{n+1}-\mathfrak{N}_{k} P}{\mathfrak{N}_{k} P^{n+1}-1}
$$

for a given ideal $B$ of $\mathcal{O}_{k}$ dividing $D$. Clearly,

$$
S(D, B)=\prod_{P \mid B} \frac{\mathfrak{N}_{k} P^{n+1}-\mathfrak{N}_{k} P}{\mathfrak{N}_{k} P^{n+1}-1} \sum_{A \mid B^{-1} D} f(A),
$$

where

$$
f(A):=\frac{\mu_{k}(A)}{\mathfrak{N}_{k} A} \prod_{\substack{P \mid A \\ P \nmid B}} \frac{\mathfrak{N}_{k} P^{n+1}-\mathfrak{N}_{k} P}{\mathfrak{N}_{k} P^{n+1}-1} .
$$

The function $f$ is multiplicative and $f\left(\mathcal{O}_{k}\right)=1$. For any prime ideal $P$ dividing $B^{-1} D$, we have

$$
f(P)= \begin{cases}-\mathfrak{N}_{k} P^{-1} & \text { if } P \mid B, \\ -\left(\mathfrak{N}_{k} P^{n}-1\right) /\left(\mathfrak{N}_{k} P^{n+1}-1\right) & \text { if } P \nmid B .\end{cases}
$$

Moreover, $f\left(P^{e}\right)=0$ if $e>1$. We use

$$
\sum_{A \mid B^{-1} D} f(A)=\prod_{P \mid B^{-1} D}(1+f(P))
$$

to obtain

$$
\begin{aligned}
S(D, B) & =\prod_{P \mid B} \frac{\mathfrak{N}_{k} P^{n+1}-\mathfrak{N}_{k} P}{\mathfrak{N}_{k} P^{n+1}-1} \prod_{\substack{P \mid B^{-1} D \\
P \nmid B}} \frac{\mathfrak{N} P^{n+1}-\mathfrak{N}_{k} P^{n}}{\mathfrak{N}_{k} P^{n+1}-1} \prod_{P \mid\left(B^{-1} D, B\right)} \frac{\mathfrak{N}_{k} P-1}{\mathfrak{N}_{k} P} \\
& =\prod_{P \mid D} \frac{\mathfrak{N}_{k} P^{n+1}-\mathfrak{N}_{k} P^{n}}{\mathfrak{N}_{k} P^{n+1}-1} \prod_{P \mid B} \frac{\mathfrak{N}_{k} P^{n+1}-\mathfrak{N}_{k} P}{\mathfrak{N}_{k} P^{n+1}-\mathfrak{N}_{k} P^{n}} \prod_{P \mid\left(B^{-1} D, B\right)} \frac{\mathfrak{N}_{k} P-1}{\mathfrak{N}_{k} P} .
\end{aligned}
$$

Let $T(D, B):=S(D, B) / \prod_{P \mid D} \frac{\mathfrak{N}_{k} P^{n+1}-\mathfrak{N}_{k} P^{n}}{\mathfrak{N}_{k} P^{n+1}-1}$. Then the expression on the lefthand side of the Lemma is given by

$$
\left(\prod_{P \mid D} \frac{\mathfrak{N}_{k} P^{n+1}-\mathfrak{N}_{k} P^{n}}{\mathfrak{N}_{k} P^{n+1}-1}\right) \sum_{B \mid D} q(\mathfrak{D}, B) T(D, B) .
$$


Since both $T(D, B)$ and $q(\mathfrak{D}, B)$ are multiplicative in $B$, this is equal to

$$
\prod_{P \mid D}\left(\frac{\mathfrak{N}_{k} P^{n+1}-\mathfrak{N}_{k} P^{n}}{\mathfrak{N}_{k} P^{n+1}-1} \sum_{j=0}^{v_{P}(D)} q\left(\mathfrak{D}, P^{j}\right) T\left(D, P^{j}\right)\right) .
$$

Elementary manipulations show that

$$
T\left(D, P^{j}\right)=\frac{\left(\mathfrak{N}_{k} P^{n}-1\right)\left(\mathfrak{N}_{k} P-1\right)}{\mathfrak{N}_{k} P^{n+1}-\mathfrak{N}_{k} P^{n}} \cdot \begin{cases}\frac{\mathfrak{N}_{k} P^{n+1}-\mathfrak{N}_{k} P^{n}}{\left(\mathfrak{N}_{k} P^{n}-1\right)\left(\mathfrak{N}_{k} P-1\right)} & \text { if } j=0, \\ 1 & \text { if } 1 \leq j<v_{P}(D), \\ \sum_{j=v_{P}(D)}^{\infty} \mathfrak{N}_{k} P^{v_{P}(D)-j} & \text { if } j=v_{P}(D) .\end{cases}
$$

Using (2.1), this shows that each of the factors in (2.2) has the form

$$
\frac{\left(\mathfrak{N}_{k} P-1\right)\left(\mathfrak{N}_{k} P^{n}-1\right)}{\mathfrak{N}_{k} P^{n+1}-1}\left(\frac{\mathfrak{N}_{k} P^{n+1}-\mathfrak{N}_{k} P^{n}}{\left(\mathfrak{N}_{k} P^{n}-1\right)\left(\mathfrak{N}_{k} P-1\right)}+\sum_{j=1}^{\infty} q\left(\mathfrak{D}, P^{j}\right)\right)=g_{P}(\mathfrak{D}, n) .
$$

Lemma 2.1 with $\mathfrak{D}:=\alpha \theta \mathcal{O}_{K}$ yields the following formula for $g_{k}(\theta, n)$.

Proposition 2.2. If $\alpha$ is nonzero and in $\mathcal{O}_{k}$ with $\alpha \theta \in \mathcal{O}_{K}$ then

$$
g_{k}(\theta, n)=\frac{V}{\mathfrak{N}_{k}(\alpha)^{n}} \prod_{P} g_{P}\left(\alpha \theta \mathcal{O}_{K}, n\right) .
$$

The next lemma shows that $g_{k}(\theta, n)$ does not depend on the choice of $\alpha$.

Lemma 2.3. Let $A$ be a nonzero ideal of $\mathcal{O}_{k}$ and $\mathfrak{D}$ a nonzero ideal of $\mathcal{O}_{K}$. Then

$$
g_{P}\left({ }^{\mathfrak{u}} A \mathfrak{D}, n\right)=\mathfrak{N}_{k} P^{n v_{P}(A)} g_{P}(\mathfrak{D}, n) .
$$

Proof. We have

$$
q\left({ }^{\mathfrak{u}} A \mathfrak{D}, P^{j}\right)= \begin{cases}\mathfrak{N}_{k} P^{n j} & \text { if } 0 \leq j<v_{P}(A), \\ \mathfrak{N}_{k} P^{n v_{P}(A)} q\left(\mathfrak{D}, P^{j-v_{P}(A)}\right) & \text { if } j \geq v_{P}(A) .\end{cases}
$$

The lemma follows by inserting these expressions for $q\left({ }^{\mathfrak{u}} A \mathfrak{D}, P^{j}\right)$ in the definition of $g_{P}\left({ }^{\mathfrak{u}} A \mathfrak{D}, n\right)$. 
Given nonzero $\alpha, \beta \in \mathcal{O}_{k}$ such that $\alpha \theta, \beta \theta \in \mathcal{O}_{K}$, then we have

$$
\mathfrak{N}_{k}(\alpha)^{n} \prod_{P} g_{P}\left(\beta \theta \mathcal{O}_{K}, n\right)=\prod_{P} g_{P}\left(\alpha \beta \theta \mathcal{O}_{K}, n\right)=\mathfrak{N}_{k}(\beta)^{n} \prod_{P} g_{P}\left(\alpha \theta \mathcal{O}_{K}, n\right),
$$

which shows the independence of $g_{k}(\theta, n)$ from the choice of $\alpha$.

To see invariance property (1.5) directly from (2.3), we need the following lemma.

Lemma 2.4. Let $\alpha \in k^{*}$. Then

$$
V(\alpha \theta, k, n)=\frac{V(\theta, k, n)}{\mathfrak{N}_{k}(\alpha)^{n}} .
$$

Proof. For any Archimedean place $v$ of $k$, the map $\phi_{v}: k_{v}^{n+1} \rightarrow k_{v}^{n+1}$ defined by $\phi_{v}\left(z_{0}, z_{1} \ldots, z_{n}\right)=\left(z_{0},|\alpha|_{v} z_{1}, \ldots,|\alpha|_{v} z_{n}\right)$ is a linear automorphism of $k_{v}^{n+1}$ (considered as $\left.\mathbb{R}^{\left[k_{v}: \mathbb{R}\right](n+1)}\right)$ of determinant $|\alpha|_{v}^{\left[k_{v}: \mathbb{R}\right] n}$. Therefore, $|\alpha|_{v}^{\left[k_{v}: \mathbb{R}\right] n} V_{v}(\alpha \theta, k, n)=$ $V_{v}(\theta, k, n)$.

Lemmas 2.3 and 2.4 imply that

$$
g_{k}(\alpha \theta, n)=g_{k}(\theta, n)
$$

for every nonzero $\alpha \in \mathcal{O}_{k}$, and hence for every $\alpha \in k^{*}$. In particular, it suffices to prove Theorem 1.2 and Theorem 1.4 for integral $\theta$.

\section{Proof of Theorem 1.2}

We start off by estimating the volume $V(\theta, k, n)$.

Lemma 3.1. We have

$$
V(\theta, k, n) \leq \mathfrak{N}_{K}(\theta)^{-n /[K: k]} .
$$

Moreover, equality holds if and only if for every Archimedean place $v$ of $k$ the absolute values $|\theta|_{w}$ are equal for all $w \mid v$.

Proof. Let $v$ be an Archimedean place of $K$, and let $p_{v}=p_{v}(\theta):=\prod_{w \mid v}|\theta|^{\frac{\left[K_{w}: k_{v}\right]}{[K: k]}}$. Consider the functions $f_{v}^{(1)}, f_{v}^{(2)}: k_{v}^{n+1} \rightarrow \mathbb{R}$ given by

$$
\begin{aligned}
f_{v}^{(1)}\left(z_{0}, \ldots, z_{n}\right) & :=\prod_{w \mid v} \max \left\{\left|z_{0}\right|_{v},|\theta|_{w}\left|z_{1}\right|_{v}, \ldots,|\theta|_{w}\left|z_{n}\right|_{v}\right\}^{\frac{\left[K K_{w}: k_{v}\right]}{\mid K: k]}}, \\
f_{v}^{(2)}\left(z_{0}, \ldots, z_{n}\right) & :=\max \left\{\left|z_{0}\right|_{v}, p_{v}\left|z_{1}\right|_{v}, \ldots, p_{v}\left|z_{n}\right|_{v}\right\} .
\end{aligned}
$$


Then $f_{v}^{(i)}(t \mathbf{z})=|t|_{v} f_{v}^{(i)}(\mathbf{z})$ holds for all $t \in k_{v}, \mathbf{z} \in k_{v}^{n+1}$, and $i \in\{1,2\}$. Moreover, $f_{v}^{(1)} \geq f_{v}^{(2)}$ as functions on $k_{v}^{n+1}$, with equality if and only if $|\theta|_{w}$ is constant on $w \mid v$.

Now $\operatorname{Vol}\left\{\mathbf{z} \in k_{v}^{n+1}: f_{v}^{(1)}(\mathbf{z})<1\right\} \leq \operatorname{Vol}\left\{\mathbf{z} \in k_{v}^{n+1}: f_{v}^{(2)}(\mathbf{z})<1\right\}$, with equality if and only if $f_{v}^{(1)}=f_{v}^{(2)}$. Evaluating both volumes gives

$$
V_{v} \leq p_{v}^{-n\left[k_{v}: \mathbb{R}\right]} \cdot \begin{cases}2^{n+1} & \text { if } v \text { is real, } \\ \pi^{n+1} & \text { if } v \text { is complex }\end{cases}
$$

with equality if and only if $|\theta|_{w}$ is constant on $w \mid v$. Thus,

$$
V(\theta, k, n) \leq \prod_{w \mid \infty}|\theta|^{-\frac{n\left[K_{w}: \mathbb{R}\right]}{[K: k]}}=\mathfrak{N}_{K}(\theta)^{-n /[K: k]},
$$

with equality if and only if the condition in the lemma is satisfied.

Let us recall some simple facts, which will be used in the sequel without further notice. Let $A, B$ be ideals of $\mathcal{O}_{k}$, and let $\mathfrak{A}, \mathfrak{B}$ be ideals of $\mathcal{O}_{K}$. Moreover, suppose that $P$ is a prime ideal of $\mathcal{O}_{k}$ and that $\mathfrak{P}$ runs over all prime ideals of $\mathcal{O}_{K}$ above $P$. Then:

- $v_{P}\left({ }^{\mathfrak{d}} \mathfrak{A}\right)=\max _{\mathfrak{P} \mid P}\left\{\left\lceil v_{\mathfrak{P}}(\mathfrak{A}) / e_{\mathfrak{P}}\right\rceil\right\}$,

- ${ }^{\mathfrak{d u}} A=A$,

- $\mathfrak{A} \mid{ }^{\mathfrak{u}} \mathfrak{A}$,

- ${ }^{\mathfrak{u}}(A B)={ }^{\mathfrak{u}} A^{\mathfrak{u}} B$,

- $\mathfrak{A} \mid{ }^{\mathfrak{u}} A$ if and only if ${ }^{\mathfrak{d}} \mathfrak{A} \mid A$.

Lemma 3.2. Let $\mathfrak{D}$ be a nonzero ideal of $\mathcal{O}_{K}$ and $P$ a prime ideal of $\mathcal{O}_{k}$. Then

$$
g_{P}(\mathfrak{D}, n) \leq \mathfrak{N}_{K}\left(\mathfrak{D}_{P}\right)^{n /[K: k]},
$$

with equality if and only if $\mathfrak{D}_{P}={ }^{\mathfrak{u}} \mathfrak{D} \mathfrak{D}_{P}$.

Proof. Lemma 2.3 and the fact that $g_{P}(\mathfrak{D}, n)=g_{P}\left(\mathfrak{D}_{P}, n\right)$ imply equality if $\mathfrak{D}_{P}={ }^{\mathfrak{u} \mathfrak{d}} \mathfrak{D}_{P}$. Therefore, let us assume that $v_{P}\left({ }^{\mathfrak{d}} \mathfrak{D}\right)=: l \geq 1$ and that $\mathfrak{D}_{P}$ is a proper divisor of ${ }^{\mathfrak{u} d} \mathfrak{D}_{P}={ }^{\mathfrak{u}} P^{l}$. Again by Lemma 2.3 , we may assume that ${ }^{\mathfrak{u}} P \nmid \mathfrak{D}$.

Let

$$
u:=\frac{1}{[K: k]} \sum_{\mathfrak{P} \mid P} f_{\mathfrak{P}} v_{\mathfrak{P}}(\mathfrak{D}),
$$

where the sum runs over all prime ideals $\mathfrak{P}$ of $\mathcal{O}_{K}$ lying over $P$, and $f_{\mathfrak{P}}=f_{\mathfrak{P} \mid P}=$ $\left[\mathcal{O}_{K} / \mathfrak{P}: \mathcal{O}_{k} / P\right]$ is the relative degree of $\mathfrak{P}$ over $P$. Then the right-hand side in the lemma is just $\mathfrak{N}_{k}(P)^{n u}$. Since $v_{P}\left({ }^{d} \mathfrak{D}\right)=l \geq 1$, we get $u>0$. Let $e_{\mathfrak{P}}=e_{\mathfrak{P} \mid P}$ be the ramification index of $\mathfrak{P}$ over $P$. As $\mathfrak{D}_{P}$ is a proper divisor of ${ }^{\mathfrak{u} d} \mathfrak{D}_{P}$, we conclude that $v_{\mathfrak{P}}(\mathfrak{D})<e_{\mathfrak{P}} v_{P}\left({ }^{\mathfrak{d}} \mathfrak{D}\right)$ for at least one $\mathfrak{P} \mid P$. Thus,

$$
\sum_{\mathfrak{P} \mid P} f_{\mathfrak{P}} v_{\mathfrak{P}}(\mathfrak{D})<\sum_{\mathfrak{P} \mid P} f_{\mathfrak{P}} e_{\mathfrak{P}} v_{P}\left({ }^{\mathfrak{D}} \mathfrak{D}\right)=[K: k] \cdot l,
$$


and therefore $u<l$. Similarly, we have

$$
q\left(\mathfrak{D}, P^{j}\right)=\mathfrak{N}_{k}(P)^{\frac{n+1}{[K: k]}\left(\sum_{\mathfrak{P} \mid P} f_{\mathfrak{P}} \min \left\{v_{\mathfrak{P}}(\mathfrak{D}), j e_{\mathfrak{P}}\right\}\right)-j},
$$

for any $j \geq 0$. By our assumption that ${ }^{\mathfrak{u}} P \nmid \mathfrak{D}$, we have $v_{\mathfrak{P}}(\mathfrak{D})<j e_{\mathfrak{P}}$ for some $\mathfrak{P} \mid P$ and all $j \geq 1$. Replacing all the minima in the above formula by their second arguments yields

$$
q\left(\mathfrak{D}, P^{j}\right)<\mathfrak{N}_{k} P^{j n} .
$$

Similarly, replacing the minima by their first arguments yields

$$
q\left(\mathfrak{D}, P^{j}\right) \leq \mathfrak{N}_{k}(P)^{(n+1) u-j},
$$

and the inequality is strict if and only if $j<l$. Let $1 \leq L \leq l$ be the integer with $L-1<u \leq L$. We use (3.2) for $j<L$ and (3.3) for $j \geq L$ to estimate $q\left(\mathfrak{D}, P^{j}\right)$ in the definition of $g_{P}(\mathfrak{D}, n)$. This shows that $g_{P}(\mathfrak{D}, n)$ is bounded from above by

$$
\frac{1}{\mathfrak{N}_{k} P^{n+1}-1}\left(\mathfrak{N}_{k} P^{L n+1}-\mathfrak{N}_{k} P^{L n}+\mathfrak{N}_{k} P^{(n+1) u-L+n+1}-\mathfrak{N}_{k} P^{(n+1) u-L+1}\right),
$$

with a strict inequality whenever $l>1$. To prove the lemma, it is enough to show that this expression is bounded by $\mathfrak{N}_{k} P^{n u}$ (with strict inequality if $l=1$ ). To this end, let $h$ be the function given by

$$
h(x):=x^{n u+n+1}-x^{n u}+x^{(n+1) u-L+1}-x^{(n+1) u-L+n+1}+x^{L n}-x^{L n+1} .
$$

Hence, we need to show that $h\left(\mathfrak{N}_{k} P\right) \geq 0$, with a strict inequality if $l=1$. With $\tilde{u}:=u-L+1 \in(0,1]$ and

$$
h_{1}(x):=x^{n \tilde{u}+n+1}-x^{n \tilde{u}}+x^{(n+1) \tilde{u}}-x^{(n+1) \tilde{u}+n}+x^{n}-x^{n+1},
$$

we have $h(x)=x^{n(L-1)} h_{1}(x)$. If $\tilde{u}=1$ then $h_{1}(x) \equiv 0$. We observe that $\tilde{u}=1$ is impossible if $l=1$, since $u<l$. Let us assume that $0<\tilde{u}<1$ and prove that, in this case, $h_{1}(x)>0$ for all $x>1$.

The function $h_{1}(x)$ is in fact a polynomial in $x^{1 /[K: k]}$. We have

$$
n \tilde{u}+n+1>\left\{\begin{array}{l}
(n+1) \tilde{u}+n \\
n+1
\end{array}>\left\{\begin{array}{l}
(n+1) \tilde{u} \\
n
\end{array}>n \tilde{u} .\right.\right.
$$

By Descartes' rule of signs, $h_{1}(x)$ has at most three positive zeros (with multiplicities). Since $h_{1}(1)=h_{1}^{\prime}(1)=h_{1}^{\prime \prime}(1)=0$ and $\lim _{x \rightarrow \infty} h_{1}(x)=\infty$, we have $h_{1}(x)>0$ for $x>1$.

We can now easily finish the proof of Theorem 1.2. After multiplying with a suitable element from $k^{*}$ we can assume that $\theta$ is an algebraic integer and choose $\alpha:=1$. From Proposition 2.2, Lemmata 3.1 and 3.2, and the observation that

$$
\mathfrak{N}_{K}(\theta)^{\frac{n}{[K: k]}}=\prod_{P} \mathfrak{N}_{K}\left(\left(\theta \mathcal{O}_{K}\right)_{P}\right)^{\frac{n}{[K: k]}},
$$


we immediately get that $g_{k}(\theta, n) \leq 1$. Now $g_{k}(\theta, n)=1$ holds if and only if we have equality in Lemmata 3.1 and 3.2. This is the case if and only if $\theta \mathcal{O}_{K}={ }^{\mathfrak{u} \mathfrak{d}} \theta \mathcal{O}_{K}$ and for each Archimedean place $v$ of $k$ the $|\theta|_{w}$ for $w \mid v$ are all equal. The condition for equality in Theorem 1.2 is just a uniform reformulation of these two statements.

\section{Preliminaries on lattices}

In this section we establish a basic counting result for lattice points, which will be used in the proofs of Theorem 6.1 and Theorem 1.3.

For a vector $\mathbf{x}$ in $\mathbb{R}^{m}$ we write $|\mathbf{x}|$ for the Euclidean length of $\mathbf{x}$. For a lattice $\Lambda$ in $\mathbb{R}^{m}$ we write $\lambda_{i}=\lambda_{i}(\Lambda)(1 \leq i \leq m)$ for the successive minima of $\Lambda$ with respect to the Euclidean distance.

Definition 4.1. Let $M$ and $m>1$ be positive integers and let $L$ be a non-negative real. We say that a set $S$ is $\operatorname{in} \operatorname{Lip}(m, M, L)$ if $S$ is a subset of $\mathbb{R}^{m}$, and if there are $M$ maps $\phi_{1}, \ldots, \phi_{M}:[0,1]^{m-1} \longrightarrow \mathbb{R}^{m}$ satisfying a Lipschitz condition

$$
\left|\phi_{i}(\mathbf{x})-\phi_{i}(\mathbf{y})\right| \leq L|\mathbf{x}-\mathbf{y}| \text { for } \mathbf{x}, \mathbf{y} \in[0,1]^{m-1}, i=1, \ldots, M,
$$

such that $S$ is covered by the images of the maps $\phi_{i}$.

We can now state and prove our counting result.

Lemma 4.2. Let $m>1$ be an integer, let $\Lambda$ be a lattice in $\mathbb{R}^{m}$ with successive minima $\lambda_{1}, \ldots, \lambda_{m}$, and let $a \in\{1, \ldots, m\}$. Let $S$ be a bounded set in $\mathbb{R}^{m}$ such that the boundary $\partial S$ of $S$ is in $\operatorname{Lip}(m, M, L), S$ is contained in the zero-centered ball of radius $L$, and $\mathbf{0} \notin S$. Then $S$ is measurable and we have

$$
|| S \cap \Lambda\left|-\frac{\operatorname{Vol} S}{\operatorname{det} \Lambda}\right| \leq c_{1}(m) M \max \left\{\frac{L^{a-1}}{\lambda_{1}{ }^{a-1}}, \frac{L^{m-1}}{\lambda_{1}^{a-1} \lambda_{a}{ }^{m-a}}\right\} .
$$

The constant $c_{1}(m)$ depends only on $m$.

Proof. Applying [19, Theorem 5.4] proves measurability and gives

$$
|| S \cap \Lambda\left|-\frac{\operatorname{Vol} S}{\operatorname{det} \Lambda}\right| \leq c_{1}(m) M \max _{0 \leq i \leq m-1} \frac{L^{i}}{\lambda_{1} \cdots \lambda_{i}} .
$$

First we assume $L / \lambda_{1} \geq 1$.

Then we conclude

$$
\begin{aligned}
\max _{0 \leq i \leq m-1} \frac{L^{i}}{\lambda_{1} \cdots \lambda_{i}} & \leq \max _{0 \leq i \leq m-a} \frac{L^{a-1}}{\lambda_{1}^{a-1}}\left(\frac{L}{\lambda_{a}}\right)^{i} \\
& =\frac{L^{a-1}}{\lambda_{1}^{a-1}} \max \left\{1, \frac{L^{m-a}}{\lambda_{a}^{m-a}}\right\} \\
& =\max \left\{\frac{L^{a-1}}{\lambda_{1}^{a-1}}, \frac{L^{m-1}}{\lambda_{1}^{a-1} \lambda_{a}{ }^{m-a}}\right\} .
\end{aligned}
$$


Next we assume $L / \lambda_{1}<1$. Then we have $|S \cap \Lambda|=0$. Moreover, by Minkowski's second theorem,

$$
\frac{\operatorname{Vol} S}{\operatorname{det} \Lambda} \leq c_{1}(m) \frac{L^{m}}{\lambda_{1} \cdots \lambda_{m}}
$$

Furthermore,

$$
\frac{L^{m}}{\lambda_{1} \cdots \lambda_{m}} \leq \frac{L^{m}}{\lambda_{1} \cdots \lambda_{m}} \frac{\lambda_{1}}{L}=\frac{L^{m-1}}{\lambda_{2} \cdots \lambda_{m}} \leq \max \left\{\frac{L^{a-1}}{\lambda_{1}{ }^{a-1}}, \frac{L^{m-1}}{\lambda_{1}{ }^{a-1} \lambda_{a}{ }^{m-a}}\right\} .
$$

We recall the following lemma, which is a special case of [3, Lemma VIII.1].

Lemma 4.3. Let $\Lambda$ be a lattice in $\mathbb{R}^{m}$. Then there exist linearly independent vectors $v_{1}, \ldots, v_{m}$ in $\Lambda$ such that $\left|v_{i}\right|=\lambda_{i}$ for $1 \leq i \leq m$.

Lemma 4.4. Let $\Lambda$ be a lattice in $\mathbb{R}^{m}$. Then there exists a basis $u_{1}, \ldots, u_{m}$ of $\Lambda$ such that

$$
\left|u_{i}\right| \leq C_{0}(m) \lambda_{1}^{-m+1} \operatorname{det} \Lambda \quad \text { for } 1 \leq i \leq m,
$$

where $C_{0}(m)$ is an explicit constant depending only on $m$.

Proof. Let $v_{1}, \ldots, v_{m}$ be linearly independent vectors as in Lemma 4.3. By a lemma of Mahler and Weyl (see [3, Lemma 8, page 135]) we obtain a basis $u_{1}, \ldots, u_{m}$ of $\Lambda$ such that $\left|u_{i}\right| \leq \max \{1, m / 2\} \lambda_{i}$. Observing that $\left|u_{i}\right| \leq\left|u_{1}\right| \cdots\left|u_{m}\right| / \lambda_{1}^{m-1}$, the lemma follows from Minkowski’s second theorem. tion 9.

The following result will be used only for the proof of Theorem 1.3 in Sec-

Lemma 4.5. Let $\Lambda_{1}$ and $\Lambda_{2}$ be lattices in $\mathbb{R}^{d}$, and consider the lattice $\Lambda:=\Lambda_{1} \times$ $\Lambda_{2}$ in $\mathbb{R}^{2 d}$. Then we have

$$
\begin{aligned}
\lambda_{1}(\Lambda) & =\min \left\{\lambda_{1}\left(\Lambda_{1}\right), \lambda_{1}\left(\Lambda_{2}\right)\right\}, \\
\lambda_{d+1}(\Lambda) & \geq \max \left\{\lambda_{1}\left(\Lambda_{1}\right), \lambda_{1}\left(\Lambda_{2}\right)\right\} .
\end{aligned}
$$

Proof. The first assertion is obvious. For the second assertion we choose, by Lemma 4.3, $d+1$ linearly independent elements $v_{j}=\left(w_{j}^{(1)}, w_{j}^{(2)}\right) \in \Lambda(1 \leq j \leq d+1)$ with $\left|v_{j}\right|=\lambda_{j}$. Clearly, not all of them can lie in $\mathbb{R}^{d} \times\{\mathbf{0}\}$, and similarly not all of them can lie in $\{\mathbf{0}\} \times \mathbb{R}^{d}$. Suppose $v_{j_{1}} \notin \mathbb{R}^{d} \times\{\mathbf{0}\}$ and $v_{j_{2}} \notin\{\mathbf{0}\} \times \mathbb{R}^{d}$. Hence $\left|v_{j_{1}}\right| \geq\left|w_{j_{1}}^{(2)}\right| \geq \lambda_{1}\left(\Lambda_{2}\right)$ and $\left|v_{j_{2}}\right| \geq\left|w_{j_{2}}^{(1)}\right| \geq \lambda_{1}\left(\Lambda_{1}\right)$. This proves the lemma. 


\section{Adelic Lipschitz heights}

In [9] Masser and Vaaler have introduced what one may call Lipschitz heights on $\mathbb{P}^{n}(K)$. This notion generalizes the absolute Weil height and allows so-called Lipschitz distance functions instead of just the maximum norm at the Archimedean places. Nonetheless, this notion is sometimes too rigid, as one often also needs modification at a finite number of non-Archimedean places. This leads naturally to the concept of adelic Lipschitz heights, introduced in [19].

\subsection{Adelic Lipschitz systems on a number field}

Let $K$ be a number field and recall that $M_{K}$ denotes the set of places of $K$, and that for every place $w$ we have fixed a completion $K_{w}$ of $K$ at $w$. We write $d_{w}=\left[K_{w}\right.$ : $\left.\mathbb{Q}_{w}\right]$ for the local degree, where $\mathbb{Q}_{w}$ denotes the completion of $\mathbb{Q}$ with respect to the unique place of $\mathbb{Q}$ that extends to $w$. The value set of $w, \Gamma_{w}:=\left\{|\alpha|_{w}: \alpha \in K_{w}\right\}$ is equal to $[0, \infty)$ if $w$ is Archimedean, and to

$$
\left\{0,\left(\mathfrak{N}_{K} \mathfrak{P}_{w}\right)^{0},\left(\mathfrak{N}_{K} \mathfrak{P}_{w}\right)^{ \pm 1 / d_{w}},\left(\mathfrak{N}_{K} \mathfrak{P}_{w}\right)^{ \pm 2 / d_{w}}, \ldots\right\}
$$

(topologized by the trivial topology) if $w$ is a non-Archimedean place corresponding to the prime ideal $\mathfrak{P}_{w}$ of $\mathcal{O}_{K}$. For $w \mid \infty$ we identify $K_{w}$ with $\mathbb{R}$ or $\mathbb{C}$, respectively, and we identify $\mathbb{C}$ with $\mathbb{R}^{2}$.

Definition 5.1. An adelic Lipschitz system $\mathcal{N}$ on $K$ (of dimension $n$ ) is a set of continuous maps

$$
N_{w}: K_{w}^{n+1} \rightarrow \Gamma_{w} \quad w \in M_{K}
$$

such that for $w \in M_{K}$ we have:

(i) $N_{w}(\mathbf{z})=0$ if and only if $\mathbf{z}=\mathbf{0}$,

(ii) $N_{w}(a \mathbf{z})=|a|_{w} N_{w}(\mathbf{z})$ for all $a \in K_{w}$ and all $\mathbf{z} \in K_{w}^{n+1}$,

(iii) if $w \mid \infty:\left\{\mathbf{z}: N_{w}(\mathbf{z})=1\right\}$ is in $\operatorname{Lip}\left(d_{w}(n+1), M_{w}, L_{w}\right)$ for some $M_{w}, L_{w}$, (iv) if $w \nmid \infty: N_{w}\left(\mathbf{z}_{1}+\mathbf{z}_{2}\right) \leq \max \left\{N_{w}\left(\mathbf{z}_{1}\right), N_{w}\left(\mathbf{z}_{2}\right)\right\}$ for all $\mathbf{z}_{1}, \mathbf{z}_{2} \in K_{w}^{n+1}$.

Moreover, we assume that the equality of functions

$$
N_{w}(\mathbf{z})=\max \left\{\left|z_{0}\right|_{w}, \ldots,\left|z_{n}\right|_{w}\right\}
$$

holds for all but a finite number of $w \in M_{K}$.

If we consider only the functions $N_{w}$ for $w \mid \infty$ then we get a Lipschitz system (of dimension $n$ ) in the sense of Masser and Vaaler [9].

For all $w \in M_{K}$ there are $c_{w} \leq 1$ in the value group $\Gamma_{w}^{*}=\Gamma_{w} \backslash\{0\}$ with

$$
c_{w} \max \left\{\left|z_{0}\right|_{w}, \ldots,\left|z_{n}\right|_{w}\right\} \leq N_{w}(\mathbf{z}) \leq c_{w}^{-1} \max \left\{\left|z_{0}\right|_{w}, \ldots,\left|z_{n}\right|_{w}\right\}
$$


for all $\mathbf{z}=\left(z_{0}, \ldots, z_{n}\right)$ in $K_{w}^{n+1}$. Due to (5.2) we can and will assume that

$$
c_{w}=1
$$

for all but a finite number of places $w$. We define

$$
C_{\mathcal{N}}^{\mathrm{fin}}:=\prod_{w \nmid \infty} c_{w}^{-\frac{d w}{[K: \mathbb{Q}]}} \geq 1,
$$

and

$$
C_{\mathcal{N}}^{\text {inf }}:=\max _{w \mid \infty}\left\{c_{w}^{-1}\right\} \geq 1 .
$$

For a prime ideal $\mathfrak{P}$ of $\mathcal{O}_{K}$ we write $v_{\mathfrak{P}}$ for the corresponding valuation on $K$, normalized by $v_{\mathfrak{P}}\left(K^{*}\right)=\mathbb{Z}$. For a nonzero fractional ideal $\mathfrak{A}$ of $K$ and a nonArchimedean place $w$ of $K$, associated to the prime $\mathfrak{P}$, we define

$$
|\mathfrak{A}|_{w}:=\mathfrak{N}_{K}(\mathfrak{P})^{-v_{\mathfrak{P}}(\mathfrak{R}) / d_{w}},
$$

so that $|\alpha|_{w}=\left|\alpha \mathcal{O}_{K}\right|_{w}$ for $\alpha \in K^{*}$. For $w \in M_{K}$ let $\sigma_{w}$ be the canonical embedding of $K$ in $K_{w}$, extended component-wise to $K^{n+1}$. For any nonzero $\omega \in K^{n+1}$, let $i_{\mathcal{N}}(\omega)$ be the unique fractional ideal of $K$ defined by

$$
N_{w}\left(\sigma_{w} \boldsymbol{\omega}\right)=\left|i_{\mathcal{N}}(\boldsymbol{\omega})\right|_{w}
$$

for all non-Archimedean $w \in M_{K}$, and we set by convention $i_{\mathcal{N}}(\mathbf{0}):=\{0\}$.

Moreover, set

$$
\mathcal{O}_{K}(\boldsymbol{\omega}):=\omega_{0} \mathcal{O}_{K}+\cdots+\omega_{n} \mathcal{O}_{K},
$$

so that $\mathcal{O}_{K}(\omega)$ is simply $i_{\mathcal{N}}(\omega)$ for any adelic Lipschitz system with (5.2) for all finite places. Now by (5.3) we get

$$
c_{w} \max \left\{\left|\omega_{0}\right|_{w}, \ldots,\left|\omega_{n}\right|_{w}\right\} \leq\left|i_{\mathcal{N}}(\boldsymbol{\omega})\right|_{w} \leq c_{w}^{-1} \max \left\{\left|\omega_{0}\right|_{w}, \ldots,\left|\omega_{n}\right|_{w}\right\} .
$$

Recall that $c_{w}=1$ up to finitely many exceptions and let

$F_{\mathcal{N}}:=\left\{\mathfrak{A}: \mathfrak{A}\right.$ nonzero fractional ideal of $K$ and $c_{w} \leq|\mathfrak{A}|_{w} \leq c_{w}^{-1}$ for all $\left.w \nmid \infty\right\}$.

By unique factorization of fractional ideals, $F_{\mathcal{N}}$ is finite. Moreover, for any $\omega \in$ $K^{n+1}$, we have

$$
i_{\mathcal{N}}(\omega)=\mathcal{O}_{K}(\omega) \mathfrak{F}(\omega)
$$

for some $\mathfrak{F}(\boldsymbol{\omega}) \in F_{\mathcal{N}}$. Taking the product in (5.7) over all finite places with multiplicities $d_{w}$ shows that

$$
C_{\mathcal{N}}^{\mathrm{fin}-[K: \mathbb{Q}]} \mathfrak{N}_{K} \mathcal{O}_{K}(\boldsymbol{\omega}) \leq \mathfrak{N}_{K} i_{\mathcal{N}}(\boldsymbol{\omega}) \leq C_{\mathcal{N}}^{\mathrm{fin}}{ }^{[K: \mathbb{Q}]} \mathfrak{N}_{K} \mathcal{O}_{K}(\boldsymbol{\omega})
$$




\subsection{Adelic Lipschitz heights on $\mathbb{P}^{n}(K)$}

Let $\mathcal{N}$ be an adelic Lipschitz system on $K$ of dimension $n$. Then the height $H_{\mathcal{N}}$ on $K^{n+1}$ is defined by

$$
H_{\mathcal{N}}(\boldsymbol{\omega}):=\prod_{w \in M_{K}} N_{w}\left(\sigma_{w}(\boldsymbol{\omega})\right)^{\frac{d_{w}}{[K: \mathbb{Q}]}} .
$$

Thanks to the product formula and (ii) from Definition $5.1 H_{\mathcal{N}}(\boldsymbol{\omega})$ is invariant under scalar multiplication by elements of $K^{*}$. Therefore $H_{\mathcal{N}}$ is well-defined on $\mathbb{P}^{n}(K)$ by setting

$$
H_{\mathcal{N}}(P):=H_{\mathcal{N}}(\omega),
$$

where $P=\left(\omega_{0}: \cdots: \omega_{n}\right) \in \mathbb{P}^{n}(K)$ and $\omega=\left(\omega_{0}, \ldots, \omega_{n}\right) \in K^{n+1}$. We note that by (5.3), (5.5) and (5.6) we have

$$
\left(C_{\mathcal{N}}^{\mathrm{fin}} C_{\mathcal{N}}^{\mathrm{inf}}\right)^{-1} H(P) \leq H_{\mathcal{N}}(P) \leq C_{\mathcal{N}}^{\mathrm{fin}} C_{\mathcal{N}}^{\mathrm{inf}} H(P),
$$

where $H(P)$ denotes the projective absolute multiplicative Weil height of $P$. Hence, by Northcott's theorem, $\left\{P \in \mathbb{P}^{n}(K): H_{\mathcal{N}}(P) \leq X\right\}$ is a finite set for each $X$ in $[0, \infty)$.

\section{The general theorem}

Let $k \subseteq K$ be number fields and let $\mathcal{N}$ be an adelic Lipschitz system of dimension $n$ on $K$. Recall that the functions $N_{w}, n$, and $K$ are all part of the data of $\mathcal{N}$. From $\mathcal{N}$ we obtain an adelic Lipschitz height $H_{\mathcal{N}}$ on $\mathbb{P}^{n}(K)$. Our goal in this section is to derive an asymptotic formula for the counting function

$$
N_{\mathcal{N}}\left(\mathbb{P}^{n}(k), X\right):=\left|\left\{P \in \mathbb{P}^{n}(k): H_{\mathcal{N}}(P) \leq X\right\}\right| .
$$

Let us set some necessary notation first. For each Archimedean place $v$ of $k$ we define a function $N_{v}$ on $k_{v}^{n+1}$ by

$$
N_{v}(\mathbf{z}):=\prod_{w \mid v} N_{w}(\mathbf{z})^{\frac{d_{w}}{d_{v}[K: k]}} .
$$

Let $\mathcal{N}^{\prime}=\mathcal{N}^{\prime}(\mathcal{N}, k)$ be the collection of functions $N_{v}$, where $N_{v}$ is as in (6.1) if $v$ is an Archimedean place of $k$ and

$$
N_{v}(\mathbf{z}):=\max \left\{\left|z_{0}\right|_{v}, \ldots,\left|z_{n}\right|_{v}\right\}
$$

if $v$ is a non-Archimedean place of $k$. From now on we assume that $\mathcal{N}^{\prime}$ is an adelic Lipschitz system (of dimension $n$ ) on $k$ (the conditions (i), (ii) and (iv) are 
automatically satisfied but (iii) may possibly fail). Hence there exists a positive integer $M_{\mathcal{N}^{\prime}}$ and a positive real number $L_{\mathcal{N}^{\prime}}$ such that the sets defined by $N_{v}(\mathbf{z})=1$ lie in $\operatorname{Lip}\left(d_{v}(n+1), M_{\mathcal{N}^{\prime}}, L_{\mathcal{N}^{\prime}}\right)$ for all Archimedean places $v$ of $k$. The sets defined by $N_{v}(\mathbf{z})<1$ are measurable and have a finite, positive volume, which we denote by $V_{v}$, and set

$$
V_{\mathcal{N}^{\prime}}:=\prod_{v \mid \infty} V_{v}
$$

We denote by $\sigma_{1}, \ldots, \sigma_{d}$ the embeddings from $k$ to $\mathbb{R}$ or $\mathbb{C}$ respectively, ordered such that $\sigma_{r+s+i}=\bar{\sigma}_{r+i}$ for $1 \leq i \leq s$. We define

$$
\begin{aligned}
& \sigma: k \longrightarrow \mathbb{R}^{r} \times \mathbb{C}^{s}=\mathbb{R}^{d} \\
& \sigma(\omega)=\left(\sigma_{1}(\omega), \ldots, \sigma_{r+s}(\omega)\right)
\end{aligned}
$$

and extend $\sigma$ component-wise to get a map

$$
\sigma: k^{n+1} \longrightarrow \mathbb{R}^{m},
$$

where $m=d(n+1)$.

For nonzero fractional ideals $C$ of $k$, and $\mathfrak{D}$ of $K$, we define the following subsets of $\mathbb{R}^{m}$ :

$$
\begin{aligned}
\Lambda_{C}^{*}(\mathfrak{D}): & =\left\{\sigma(\boldsymbol{\omega}): \boldsymbol{\omega} \in k^{n+1}, \mathcal{O}_{k}(\boldsymbol{\omega})=C, i_{\mathcal{N}}(\boldsymbol{\omega})=\mathfrak{D}\right\}, \\
\Lambda_{C}(\mathfrak{D}): & =\left\{\sigma(\boldsymbol{\omega}): \boldsymbol{\omega} \in k^{n+1}, \mathcal{O}_{k}(\boldsymbol{\omega})=C, i_{\mathcal{N}}(\boldsymbol{\omega}) \subseteq \mathfrak{D}\right\}, \\
\Lambda(\mathfrak{D}): & =\left\{\sigma(\boldsymbol{\omega}): \boldsymbol{\omega} \in k^{n+1}, i_{\mathcal{N}}(\boldsymbol{\omega}) \subseteq \mathfrak{D}\right\} .
\end{aligned}
$$

Note that by (5.8) we have

$$
\mathfrak{D} \in{ }^{\mathfrak{u}} C F_{\mathcal{N}}
$$

whenever $\Lambda_{C}^{*}(\mathfrak{D})$ is non-empty, where ${ }^{\mathfrak{u}} C F_{\mathcal{N}}$ denotes the finite set of fractional ideals of the form ${ }^{\mathfrak{u}} C \mathfrak{F}$ with $\mathfrak{F} \in F_{\mathcal{N}}$.

Let $\mathcal{R}$ be a set of integral representatives for the class group $\mathrm{Cl}_{k}$. For any $C \in \mathcal{R}$, we choose a finite set $S_{C}$ of nonzero fractional ideals of $K$ such that

$$
S_{C} \text { contains all } \mathfrak{D} \text { with } \Lambda_{C}^{*}(\mathfrak{D}) \neq \emptyset .
$$

Moreover, we choose a finite set $T$ in the following way. For any $\mathfrak{D} \in S_{C}$, let $T_{C, \mathfrak{D}}$ be the set of all nonzero ideals $\mathfrak{A}$ of $\mathcal{O}_{K}$ such that $\Lambda_{C}(\mathfrak{A D}) \neq \emptyset$. This set is finite, since, similar as above, we have $\mathfrak{A D E} \in{ }^{\mathfrak{u}} C F_{\mathcal{N}}$ for some ideal $\mathfrak{E}$ of $\mathcal{O}_{K}$ whenever $\Lambda_{C}(\mathfrak{A D}) \neq \emptyset$. Then we choose $T$ to be any finite set of nonzero ideals of $\mathcal{O}_{K}$ such that

$$
T \text { contains all the sets } T_{C, \mathfrak{D}} \text { for } C \in \mathcal{R} \text { and } \mathfrak{D} \in S_{C} \text {. }
$$


We define

$$
g_{k}^{\mathcal{N}}:=\sum_{C \in \mathcal{R}} \sum_{\mathfrak{D} \in S_{C}} \sum_{\mathfrak{A} \in T} \mu_{K}(\mathfrak{A}) \sum_{E \unlhd \mathcal{O}_{k}} \mu_{k}(E) \frac{\mathfrak{N}_{K} \mathfrak{D}^{\frac{n+1}{[K: k]}}}{\operatorname{det} \Lambda(\mathfrak{A D}, C E)},
$$

where

$$
\Lambda(\mathfrak{A D}, C E)=\Lambda(\mathfrak{A D}) \cap \sigma\left((C E)^{n+1}\right) .
$$

Note that the infinite sum in (6.6) taken over all nonzero ideals $E$ converges absolutely, as det $\Lambda(\mathfrak{A D}, C E) \geq\left(2^{-s} \mathfrak{N}_{k} C E\right)^{n+1}$. Although $g_{k}^{\mathcal{N}}$ seems to depend on the choice of $\mathcal{R}, S_{C}$ and $T$, we will see that this is actually not the case. Of course, one could impose a minimality condition to render the choice of the sets $S_{C}$ and $T$ unique, but for the calculation of $g_{k}^{\mathcal{N}}$ it is convenient to have more flexibility for the choices of these sets. From Theorem 6.1,(5.10), and Schanuel's theorem it will follow that $g_{k}^{\mathcal{N}}>0$.

Finally, we define

$$
A_{\mathcal{N}}:=A_{\mathcal{N}}(k):=\left|F_{\mathcal{N}}\right| M_{\mathcal{N}^{\prime}}^{r+s}\left(\left(L_{\mathcal{N}^{\prime}}+C_{\mathcal{N}^{\prime}}^{\text {inf }}\right) C_{\mathcal{N}}^{\text {fin }}\right)^{d(n+1)-1}
$$

We can now state the theorem.

Theorem 6.1. Let $k \subseteq K$ be number fields, $d:=[k: \mathbb{Q}]$, let $\mathcal{N}$ be an adelic Lipschitz system (of dimension $n$ ) on $K$, and suppose that $\mathcal{N}^{\prime}=\mathcal{N}^{\prime}(\mathcal{N}, k)$ is an adelic Lipschitz system (of dimension $n$ ) on $k$. Then, as $X \geq 1$ tends to infinity, we have

$$
N_{\mathcal{N}}\left(\mathbb{P}^{n}(k), X\right)=\omega_{k}^{-1}(n+1)^{r+s-1} R_{k} V_{\mathcal{N}^{\prime}} g_{k}^{\mathcal{N}} X^{d(n+1)}+O\left(|T| A_{\mathcal{N}} X^{d(n+1)-1} \mathfrak{L}\right),
$$

where $\mathfrak{L}=1+\log \left(C_{\mathcal{N}^{\prime}}^{\mathrm{inf}} C_{\mathcal{N}}^{\mathrm{fin}} X\right)$ if $(n, d)=(1,1)$ and $\mathfrak{L}=1$ otherwise. The implicit constant in the $O$-term depends only on $k$ and on $n$.

The hypothesis of $\mathcal{N}^{\prime}$ being an adelic Lipschitz system is a minor one. For instance, this hypothesis is certainly fulfilled when the functions $N_{w}$ of $\mathcal{N}$ are norms, as we shall prove in the appendix (see Lemma A.1).

\section{Proof of Theorem 6.1}

The proof of Theorem 6.1 makes frequent use of arguments from [9] and [19] (some of which can be traced back to [12], or even to Dedekind and Weber).

Let $q:=r+s-1, \Sigma$ the hyperplane in $\mathbb{R}^{q+1}$ defined by $x_{1}+\cdots+x_{q+1}=0$ and $\delta=\left(d_{1}, \ldots, d_{q+1}\right)$ with $d_{i}=1$ for $1 \leq i \leq r$ and $d_{i}=2$ for $r+1 \leq i \leq$ $r+s=q+1$. The $\operatorname{map} l(\eta):=\left(d_{1} \log \left|\sigma_{1}(\eta)\right|, \ldots, d_{q+1} \log \left|\sigma_{q+1}(\eta)\right|\right)$ sends $k^{*}$ to $\mathbb{R}^{q+1}$. For $q>0$ the image of the unit group $\mathcal{O}_{k}^{*}$ under $l$ is a lattice in $\Sigma$ with determinant $\sqrt{q+1} R_{k}$. 
We now define a set $S_{F}(t)$ using our adelic Lipschitz system $\mathcal{N}^{\prime}$ on $k$. Let $F$ be a bounded set in $\Sigma$ and for $t>0$ let $F(t)$ be the vector sum

$$
F(t):=F+\delta(-\infty, \log t] .
$$

We denote by exp the diagonal exponential map from $\mathbb{R}^{q+1}$ to $(0, \infty)^{q+1}$. Any embedding $\sigma_{i}(1 \leq i \leq q+1)$ corresponds to an Archimedean place $v$, and thus gives rise to one of our Lipschitz distance functions $N_{i}:=N_{v}$ from $\mathcal{N}^{\prime}$. We use variables $\mathbf{z}_{1}, \ldots, \mathbf{z}_{q+1}$ with $\mathbf{z}_{i}$ in $\mathbb{R}^{d_{i}(n+1)}$. Exactly as in [9] we define $S_{F}(t)$ in $\mathbb{R}^{m}$ for $m=\sum_{i=1}^{q+1} d_{i}(n+1)=d(n+1)$ as the set of all $\mathbf{z}_{1}, \ldots, \mathbf{z}_{q+1}$ such that

$$
\left(N_{1}\left(\mathbf{z}_{1}\right)^{d_{1}}, \ldots, N_{q+1}\left(\mathbf{z}_{q+1}\right)^{d_{q+1}}\right) \in \exp (F(t)) .
$$

We note that

$$
\mathbf{0} \notin S_{F}(t) .
$$

Using (ii) from Definition 5.1 it is easily seen that $S_{F}(t)$ is homogeneously expanding, i.e.,

$$
S_{F}(t)=t S_{F}(1) .
$$

Moreover, if $F$ lies in a zero-centered ball of radius $r_{F}$ then

$$
S_{F}(t) \subseteq\left\{\left(\mathbf{z}_{1}, \ldots, \mathbf{z}_{q+1}\right): N_{i}\left(\mathbf{z}_{i}\right) \leq \exp \left(r_{F}\right) t \text { for } 1 \leq i \leq q+1\right\} .
$$

The latter set lies in the the zero-centered ball of radius $\sqrt{m} C_{\mathcal{N}^{\prime}}^{\mathrm{inf}} \exp \left(r_{F}\right) t$, and thus

$$
S_{F}(t) \subseteq B_{0}\left(\sqrt{m} C_{\mathcal{N}^{\prime}}^{\mathrm{inf}} \exp \left(r_{F}\right) t\right) .
$$

Note that for $q=0$ we automatically have $F=\{0\}$, and our set $S_{F}(t)$ is precisely the set defined by $N_{1}(\mathbf{z}) \leq t$.

We now specify our set $F$ when $q>0$. We choose a basis $u_{1}, \ldots, u_{q}$ of the lattice $l\left(O_{k}^{*}\right)$ as in Lemma 4.4. Set $F:=[0,1) u_{1}+\cdots+[0,1) u_{q}$. So $F$ is measurable of $(q$-dimensional) volume

$$
\operatorname{Vol}(F)=\sqrt{q+1} R_{k}
$$

(and this remains true for $q=0$ ). From the argument in [19] following (8.2), we see that $\lambda_{1}\left(l\left(\mathcal{O}_{k}^{*}\right)\right) \geq c_{d}$ for some positive constant $c_{d}$ depending only on $d$. With the estimate from Lemma 4.4, we get

$$
\left|u_{i}\right| \leq C_{0}(q) c_{d}^{-q+1} \operatorname{Vol}(F) \leq C_{d} R_{k}, \quad(1 \leq i \leq q)
$$


for some positive constant $C_{d}$ depending only on $d$. Note that $F$ lies in the zero centered ball of radius $q C_{d} R_{k}$, and this remains trivially true for $q=0$. Therefore by (7.5)

$$
S_{F}(t) \subseteq B_{0}(\kappa t),
$$

where

$$
\kappa:=\sqrt{m} C_{\mathcal{N}^{\prime}}^{\mathrm{inf}} \exp \left(q C_{d} R_{k}\right) .
$$

Lemma 7.1. There exists a constant $c_{k}(n)$ depending only on $k$ and $n$, a positive integer $\widetilde{M}$, and a positive real $\widetilde{L}$ with $\widetilde{M} \leq c_{k}(n) M_{\mathcal{N}^{\prime}}^{q+1}, \widetilde{L} \leq c_{k}(n)\left(L_{\mathcal{N}^{\prime}}+C_{\mathcal{N}^{\prime}}^{\text {inf }}\right)$, such that

$$
\partial S_{F}(t) \in \operatorname{Lip}(m, \tilde{M}, \tilde{L} t) \text { and } S_{F}(t) \subseteq B_{0}(\widetilde{L} t) .
$$

Proof. The second part follows immediately from (7.8) and (7.9).

Let us now prove the first part. For $q=0$ our set $S_{F}(t)$ is precisely the set defined by $N_{v}(\mathbf{z}) \leq t$, where $v$ is the single Archimedean place of $k$. So the boundary of $S_{F}(t)$ is the set $\left\{\mathbf{z}: N_{v}(\mathbf{z})=t\right\}=t\left\{\mathbf{z}: N_{v}(\mathbf{z})=1\right\}$. By assumption $\mathcal{N}^{\prime}$ is an adelic Lipschitz system, and thus the latter set lies in $\operatorname{Lip}\left(m, M_{\mathcal{N}^{\prime}}, L_{\mathcal{N}^{\prime}} t\right)$. This proves the lemma for $q=0$.

Suppose now that $q \geq 1$. Then we can find $2 q$ linear maps $\psi_{i}:[0,1]^{q-1} \rightarrow \Sigma$ parameterizing $\partial F$ that, because of (7.7), will satisfy a Lipschitz condition with constant $(q-1) C_{d} R_{k}$ (for $q=1$ this is simply interpreted as $|\partial F| \leq 2$ ). The claim now follows from [19, Lemma 7.1] by a simple computation.

We conclude from [9, Lemma 4], (7.6), and (7.4) that $S_{F}(t)$ is measurable and has volume

$$
\operatorname{Vol} S_{F}(t)=(n+1)^{q} R_{k} V_{\mathcal{N}^{\prime}} t^{m} .
$$

Lemma 7.2. We have

$$
N_{\mathcal{N}}\left(\mathbb{P}^{n}(k), X\right)=\omega_{k}^{-1} \sum_{C \in \mathcal{R}} \sum_{\mathfrak{D} \in S_{C}}\left|\Lambda_{C}^{*}(\mathfrak{D}) \cap S_{F}\left(X \mathfrak{N}_{K} \mathfrak{D}^{1 /[K: \mathbb{Q}]}\right)\right| .
$$

Proof. Let $P \in \mathbb{P}^{n}(k)$ with homogeneous coordinates $\left(\omega_{0}, \ldots, \omega_{n}\right)=\omega \in k^{n+1} \backslash\{\boldsymbol{0}\}$. Recall the definition of the adelic Lipschitz system $\mathcal{N}^{\prime}$. The functions $N_{v}$ (or $N_{i}$ ) will denote those associated with $\mathcal{N}^{\prime}$, whereas $N_{w}$ will denote a function associated with the adelic Lipschitz system $\mathcal{N}$ on $K$.

Now

$$
i_{\mathcal{N}^{\prime}}(\boldsymbol{\omega})=\mathcal{O}_{k}(\boldsymbol{\omega})
$$

Suppose $\varepsilon \in k^{*}$. Then we have

$$
i_{\mathcal{N}^{\prime}}(\varepsilon \boldsymbol{\omega})=\varepsilon i_{\mathcal{N}^{\prime}}(\boldsymbol{\omega}) .
$$


Hence the ideal class of $i_{\mathcal{N}^{\prime}}(\omega)$ is independent of the coordinates $\omega$ we have chosen. In particular, we can choose $\omega$ such that $i_{\mathcal{N}^{\prime}}(\omega)=C$ for some unique $C$ in $\mathcal{R}$. Thus, $\omega$ is unique up to scalar multiplication by units $\eta$, and moreover, $i_{\mathcal{N}}(\omega):=\mathfrak{D} \in S_{C}$. The set $F(\infty)=F+\mathbb{R} \boldsymbol{\delta}$ is a fundamental set of $\mathbb{R}^{q+1}$ under the action of the additive subgroup $l\left(\mathcal{O}_{k}^{*}\right)$. Because of Definition 5.1, (ii) we have

$$
\log N_{i}\left(\sigma_{i}(\eta \omega)\right)^{d_{i}}=\log N_{i}\left(\sigma_{i} \omega\right)^{d_{i}}+d_{i} \log \left|\sigma_{i} \eta\right|
$$

for $1 \leq i \leq q+1$. Hence, there exist exactly $\omega_{k}$ representatives $\omega$ of $P$ with

$$
\left(d_{1} \log N_{1}\left(\sigma_{1} \omega\right), \ldots, d_{q+1} \log N_{q+1}\left(\sigma_{q+1} \omega\right)\right) \in F(\infty) .
$$

But the above is equivalent with

$$
\left(N_{1}\left(\sigma_{1} \omega\right)^{d_{1}}, \ldots, N_{q+1}\left(\sigma_{q+1} \omega\right)^{d_{q+1}}\right) \in \exp (F(\infty)) .
$$

Furthermore

$$
\exp \left(F\left(t_{0}\right)\right)=\left\{\left(X_{1}, \ldots, X_{q+1}\right) \in \exp (F(\infty)): X_{1} \cdots X_{q+1} \leq t_{0}^{d}\right\} .
$$

Hence, for all $\omega_{k}$ representatives $\omega$ of $P$ as above, the inequality

$$
\prod_{v \mid \infty} N_{v}\left(\sigma_{v}(\boldsymbol{\omega})\right)^{d_{v} / d}=\prod_{v \mid \infty} \prod_{w \mid v} N_{w}\left(\sigma_{w}(\boldsymbol{\omega})\right)^{d_{w} /[K: \mathbb{Q}]} \leq t_{0}
$$

is equivalent to

$$
\sigma \omega \in S_{F}\left(t_{0}\right)
$$

On the other hand,

$$
\prod_{w \nmid \infty} N_{w}\left(\sigma_{w}(\boldsymbol{\omega})\right)^{d_{w} /[K: \mathbb{Q}]}=\mathfrak{N}_{K} i_{\mathcal{N}}(\boldsymbol{\omega})^{-1 /[K: \mathbb{Q}]}=\mathfrak{N}_{K} \mathfrak{D}^{-1 /[K: \mathbb{Q}]} .
$$

As

$$
H_{\mathcal{N}}(P)=\prod_{v \mid \infty} \prod_{w \mid v} N_{w}\left(\sigma_{w}(\boldsymbol{\omega})\right)^{d_{w} /[K: \mathbb{Q}]} \prod_{w \nmid \infty} N_{w}\left(\sigma_{w}(\boldsymbol{\omega})\right)^{d_{w} /[K: \mathbb{Q}]},
$$

the claim follows.

Lemma 7.3. We have

$$
\begin{aligned}
& N_{\mathcal{N}}\left(\mathbb{P}^{n}(k), X\right) \\
& =\omega_{k}^{-1} \sum_{C \in \mathcal{R}} \sum_{\mathfrak{D} \in S_{C}} \sum_{\mathfrak{A} \in T} \mu_{K}(\mathfrak{A}) \sum_{E \unlhd \mathcal{O}_{k}} \mu_{k}(E)\left|\Lambda(\mathfrak{A} \mathfrak{D}, C E) \cap S_{F}\left(X \mathfrak{N}_{K} \mathfrak{D}^{1 /[K: \mathbb{Q}]}\right)\right|,
\end{aligned}
$$

where $E$ runs over all nonzero ideals of $\mathcal{O}_{k}$. 
Proof. We start off from Lemma 7.2 and we apply Möbius inversion twice to get rid of the two coprimality conditions $C$ and *

Directly from the definition we get

$$
\Lambda_{C}(\mathfrak{A D})=\bigcup_{\mathfrak{B}} \Lambda_{C}^{*}(\mathfrak{A} \mathfrak{B D}),
$$

where $\mathfrak{B}$ runs over all nonzero ideals of $\mathcal{O}_{K}$. This is clearly a disjoint union. Note that $\Lambda_{C}^{*}(\mathfrak{A} \mathfrak{B D}) \neq \varnothing$ only when $\mathfrak{A} \mathfrak{B D}$ lies in the finite set $S_{C}$. Möbius inversion leads then to

$$
\begin{aligned}
\left|\Lambda_{C}^{*}(\mathfrak{D}) \cap S_{F}\left(X \mathfrak{N}_{K} \mathfrak{D}^{1 /[K: \mathbb{Q}]}\right)\right| & =\sum_{\mathfrak{A}} \mu_{K}(\mathfrak{A}) \sum_{\mathfrak{B}}\left|\Lambda_{C}^{*}(\mathfrak{A} \mathfrak{B D}) \cap S_{F}\left(X \mathfrak{N}_{K} \mathfrak{D}^{1 /[K: \mathbb{Q}]}\right)\right| \\
& =\sum_{\mathfrak{A}} \mu_{K}(\mathfrak{A})\left|\Lambda_{C}(\mathfrak{A D}) \cap S_{F}\left(X \mathfrak{N}_{K} \mathfrak{D}^{1 /[K: \mathbb{Q}]}\right)\right|,
\end{aligned}
$$

where the sums run over all nonzero ideals in $\mathcal{O}_{K}$. Next note that by definition of $T_{C, \mathfrak{D}}$ we have $\Lambda_{C}(\mathfrak{A D})=\emptyset$ whenever $\mathfrak{A} \notin T_{C, \mathfrak{D}}$. As $T_{C, \mathfrak{D}} \subseteq T$ we can restrict the last sum to $\mathfrak{A} \in T$ and we get

$$
\left|\Lambda_{C}^{*}(\mathfrak{D}) \cap S_{F}\left(X \mathfrak{N}_{K} \mathfrak{D}^{1 /[K: \mathbb{Q}]}\right)\right|=\sum_{\mathfrak{A} \in T} \mu_{K}(\mathfrak{A})\left|\Lambda_{C}(\mathfrak{A D}) \cap S_{F}\left(X \mathfrak{N}_{K} \mathfrak{D}^{1 /[K: \mathbb{Q}]}\right)\right| .
$$

We now deal with the second coprimality condition ${ }_{C}$. Also directly from the definition we get

$$
\Lambda(\mathfrak{A D}, E C)=\Lambda(\mathfrak{A D}) \cap \sigma\left((E C)^{n+1}\right)=\bigcup_{B \unlhd \mathcal{O}_{k}} \Lambda_{E C B}(\mathfrak{A D}) \cup\{0\} .
$$

Again, $B$ runs over all nonzero ideals of $\mathcal{O}_{k}$ and the union is disjoint. As $\sigma\left((E C)^{n+1}\right)$ is a lattice and $S_{F}\left(X \mathfrak{N}_{K} \mathfrak{D}^{1 /[K: \mathbb{Q}]}\right)$ is bounded we conclude from the latter equality that $\Lambda_{E C B}(\mathfrak{A D}) \cap S_{F}\left(X \mathfrak{N}_{K} \mathfrak{D}^{1 /[K: \mathbb{Q}]}\right)$ is empty for all but finitely many $B$. Möbius inversion and (7.3) lead therefore to

$$
\begin{aligned}
& \left|\Lambda_{C}(\mathfrak{A D}) \cap S_{F}\left(X \mathfrak{N}_{K} \mathfrak{D}^{1 /[K: \mathbb{Q}]}\right)\right| \\
& =\sum_{E \unlhd \mathcal{O}_{k}} \mu_{k}(E) \sum_{B \unlhd \mathcal{O}_{k}}\left|\Lambda_{E C B}(\mathfrak{A D}) \cap S_{F}\left(X \mathfrak{N}_{K} \mathfrak{D}^{1 /[K: \mathbb{Q}]}\right)\right| \\
& =\sum_{E \unlhd \mathcal{O}_{k}} \mu_{k}(E)\left|\Lambda(\mathfrak{A D}, C E) \cap S_{F}\left(X \mathfrak{N}_{K} \mathfrak{D}^{1 /[K: \mathbb{Q}]}\right)\right| .
\end{aligned}
$$

In view of Lemma 7.2 this proves the claim.

We choose a positive real $\Gamma$ such that for any $C \in \mathcal{R}$ and any $\mathfrak{D} \in S_{C}$

$$
\Gamma \leq \frac{\mathfrak{N}_{k} C}{\mathfrak{N}_{K}(\mathfrak{D})^{1 /[K: k]}} .
$$


Before we proceed note that if $S_{C}$ is chosen minimal for all $C \in \mathcal{R}$ (i.e. $S_{C}=$ $\left.\left\{i_{\mathcal{N}}(\boldsymbol{\omega}): \omega \in k^{n+1}, \mathcal{O}_{k}(\boldsymbol{\omega})=C\right\}\right)$ then it follows from (5.9) that we can choose $\Gamma=C_{\mathcal{N}}^{\text {fin }}{ }^{-d}$, and moreover, $\left|S_{C}\right| \leq\left|F_{\mathcal{N}}\right|$.

Lemma 7.4. Let $\lambda_{1}=\lambda_{1}(\Lambda(\mathfrak{A} \mathfrak{D}, \underset{\sim}{C} E))$ be the first successive minimum of the lattice $\Lambda(\mathfrak{A D}, C E)$, and let $\widetilde{M}$ and $\widetilde{L}$ be as in Lemma 7.1. Then we have

$$
\begin{aligned}
\left|\Lambda(\mathfrak{A D}, C E) \cap S_{F}\left(X \mathfrak{N}_{K} \mathfrak{D}^{1 /[K: \mathbb{Q}]}\right)\right| & =\frac{\operatorname{Vol} S_{F}(1) \mathfrak{N}_{K} \mathfrak{D}^{\frac{n+1}{[K: k]}} X^{m}}{\operatorname{det} \Lambda(\mathfrak{A D}, C E)} \\
& +O\left(\tilde{M}_{K} \frac{\mathfrak{N}_{K} \mathfrak{D}^{\frac{m-1}{[K: \mathbb{Q}]}}(\widetilde{L} X)^{m-1}}{\lambda_{1}^{m-1}}\right),
\end{aligned}
$$

where the constant in the $O$-term depends only on $m$. Moreover, with $\Gamma$ as in (7.13) we have

$$
\lambda_{1} \geq \mathfrak{N}_{K}(\mathfrak{D})^{1 /[K: \mathbb{Q}]}\left(\Gamma \mathfrak{N}_{k}(E)\right)^{1 / d}
$$

And finally, with $\kappa$ as in (7.9), if $\mathfrak{N}_{k} E>(\kappa X)^{d} / \Gamma$ then

$$
\Lambda(\mathfrak{A D}, C E) \cap S_{F}\left(X \mathfrak{N}_{K} \mathfrak{D}^{1 /[K: \mathbb{Q}]}\right)=\emptyset .
$$

Proof. For the first assertion we use (7.3) and apply Lemma 4.2 with $a=m$. Thanks to (7.8) and Lemma 7.1 the required conditions are satisfied, and using (7.4) the first result drops out.

Now for the second statement we first observe that $\lambda_{1}$ is at least as large as the first successive minimum of the lattice $\sigma(C E)$. But it is well-known that the latter is at least $\mathfrak{N}_{k}(C E)^{1 / d}$, see, e.g., [9, Lemma 5]. Now as $\mathfrak{D} \in S_{C}$ and by the definition of $\Gamma$ we get $\mathfrak{N}_{k} C \geq \Gamma \mathfrak{N}_{K}(\mathfrak{D})^{1 /[K: k]}$ and this yields the second assertion.

The last claim follows upon combining the above estimate for $\lambda_{1}$ with (7.3), (7.8).

We can now conclude the proof of Theorem 6.1. Let us first assume that $(n, d) \neq$ $(1,1)$. Combining Lemma 7.3, Lemma 7.4 and (7.11) gives the main term as in Theorem 6.1. The error term is bounded by

$$
\begin{aligned}
& \sum_{C \in \mathcal{R}} \sum_{\mathfrak{D} \in S_{C}} \sum_{\mathfrak{A} \in T} \sum_{E \unlhd \mathcal{O}_{k}} O\left(\tilde{M} \frac{\mathfrak{N}_{K} \mathfrak{D}^{\frac{m-1}{K: \mathbb{Q}}}(\tilde{L} X)^{m-1}}{\lambda_{1}^{m-1}}\right) \\
\leq & \sum_{C \in \mathcal{R}} \sum_{\mathfrak{D} \in S_{C}} \sum_{\mathfrak{A} \in T} \sum_{E \unlhd \mathcal{O}_{k}} O\left(\frac{\tilde{M}(\widetilde{L} X)^{m-1}}{\Gamma^{(m-1) / d} \mathfrak{N}_{k} E^{(n+1)-1 / d}}\right) \\
\leq & \sum_{C \in \mathcal{R}} \sum_{\mathfrak{D} \in S_{C}} \sum_{\mathfrak{A} \in T} O\left(\frac{\tilde{M}(\widetilde{L} X)^{m-1}}{\Gamma^{(m-1) / d}}\right) \\
= & O\left(\sum_{C \in \mathcal{R}}\left|S_{C}\right||T| \frac{\tilde{M}(\widetilde{L} X)^{m-1}}{\Gamma^{(m-1) / d}}\right) .
\end{aligned}
$$


This proves the theorem in the case $(n, d) \neq(1,1)$ except that the constant in the error term is different from the one in the statement of the theorem. In particular, it shows that the main term is independent of the particular choice of the sets $S_{C}$. However, if we choose all the sets $S_{C}$ to be minimal then, by the remark just after (7.13), we can choose $\Gamma=C_{\mathcal{N}}^{f i n-d}$, and $\left|S_{C}\right| \leq\left|F_{\mathcal{N}}\right|$. This, and not forgetting the definition of $\widetilde{M}$ and $\widetilde{L}$ from Lemma 7.1, yields the desired error term.

We now assume $(n, d)=(1,1)$ (which of course means $k=\mathbb{Q}, \mathcal{R}=\{C\}$, $\omega_{k}=2$ ). Using also the last part of Lemma 7.4 we conclude

$$
\begin{aligned}
& N_{\mathcal{N}}\left(\mathbb{P}^{1}(\mathbb{Q}), X\right)= \\
& \frac{1}{2} \sum_{\mathfrak{D} \in S_{C}} \sum_{\mathfrak{A} \in T} \mu_{K}(\mathfrak{A}) \sum_{\substack{E \unlhd \mathbb{Z} \\
\mathfrak{N}_{\mathbb{Q}} E \leq \kappa X / \Gamma}} \mu_{\mathbb{Q}}(E)\left|\Lambda(\mathfrak{A D}, C E) \cap S_{F}\left(X \mathfrak{N}_{K} \mathfrak{D}^{1 /[K: \mathbb{Q}]}\right)\right| \\
& =\frac{1}{2} \sum_{\mathfrak{D} \in S_{C}} \sum_{\mathfrak{A} \in T} \mu_{K}(\mathfrak{A}) \sum_{E \unlhd \mathbb{Z}} \mu_{\mathbb{Q}}(E) \frac{\operatorname{Vol} S_{F}(1) \mathfrak{N}_{K} \mathfrak{D}^{\frac{2}{K: \mathbb{Q}}} X^{2}}{\operatorname{det} \Lambda(\mathfrak{A D}, C E)} \\
& +O\left(\sum_{\mathfrak{D} \in S_{C}} \sum_{\mathfrak{A} \in T} \sum_{\substack{E \unlhd \mathbb{Z} \\
\mathfrak{n}_{\mathbb{Q}} E>K X / \Gamma}} \frac{\operatorname{Vol} S_{F}(1) \mathfrak{N}_{K} \mathfrak{D} \frac{2}{[K: \mathbb{Q}]} X^{2}}{\operatorname{det} \Lambda(\mathfrak{A D}, C E)}\right) \\
& +O\left(\sum_{\mathfrak{D} \in S_{C}} \sum_{\mathfrak{A} \in T} \sum_{\substack{E \unlhd \mathbb{Z} \\
\mathfrak{M}_{\mathbb{Q}}^{E \leq \kappa X / \Gamma}}} \frac{\tilde{M} \mathfrak{N}_{K} \mathfrak{D}^{\frac{1}{K: \mathbb{Q}}} \tilde{L} X}{\lambda_{1}}\right) .
\end{aligned}
$$

Now the first term gives the main term as before. For the second term we use Minkowski's first theorem to estimate the determinant in terms of $\lambda_{1}$, and then a simple computation using Lemma 7.4 and (7.8) gives the error term $O\left(\left|S_{C}\right||T|(1+\right.$ $\kappa X / \Gamma)$. For the last error term we use again Lemma 7.4, and again a simple computation yields the error term

$$
O\left(\left|S_{C}\right||T|(\tilde{M} \tilde{L} / \Gamma) X(1+\log (\kappa X / \Gamma)) .\right.
$$

To get the right error term we choose again $S_{C}$ to be minimal so that we can take $\Gamma=C_{\mathcal{N}}^{f i n}{ }^{-1}$, and $\left|S_{C}\right| \leq\left|F_{\mathcal{N}}\right|$. This proves Theorem 6.1.

\section{Proof of Theorem 1.4}

In this section, we deduce Theorem 1.4 from Theorem 6.1. Recall the simple facts mentioned just before Lemma 3.2.

As mentioned after Lemma 2.4, we can and will assume that $\theta$ is an algebraic integer. Let $K:=k(\theta)$, and let $\mathcal{N}$ be the adelic Lipschitz system on $K$ of dimension 
$n$ defined by

$$
N_{w}(\mathbf{z}):=\max \left\{\left|z_{0}\right|_{w},|\theta|_{w}\left|z_{1}\right|_{w}, \ldots,|\theta|_{w}\left|z_{n}\right|_{w}\right\}
$$

so

$$
i_{\mathcal{N}}(\boldsymbol{\omega})=\omega_{0} \mathcal{O}_{K}+\theta \omega_{1} \mathcal{O}_{K}+\cdots+\theta \omega_{n} \mathcal{O}_{K}
$$

Lemma 8.1. We have

$$
N\left(\theta k^{n}, X\right)=N_{\mathcal{N}}\left(\mathbb{P}^{n}(k), X\right)+O\left(X^{n d}\right),
$$

where the implicit constant in the error term depends only on $k, \theta$, and $n$.

Proof. The points $\boldsymbol{\alpha}=\left(\omega_{1} / \omega_{0}, \ldots, \omega_{n} / \omega_{0}\right) \in k^{n}$ with $H(\theta \boldsymbol{\alpha}) \leq X$ are in one-toone correspondence with the projective points $P=\left(\omega_{0}: \cdots: \omega_{n}\right) \in \mathbb{P}^{n}(k)$ with $\omega_{0} \neq 0$ and $H_{\mathcal{N}}(P) \leq X$.

If $n>1$ then we can apply Theorem 6.1 with $n-1$ and the adelic Lipschitz system given by the norm functions (see Lemma A.1 in the appendix)

$$
N_{w}\left(\left(z_{1}, \ldots, z_{n}\right)\right):=\max \left\{|\theta|_{w}\left|z_{1}\right|_{w}, \ldots,|\theta|_{w}\left|z_{n}\right|_{w}\right\}
$$

(with $\mathcal{R}, S_{C}$ and $T$ chosen in such a way that $|T|$ is minimal) to see that the number of such points $P$ with $\omega_{0}=0$ is $O\left(X^{n d}\right)$. This trivially remains true for $n=1$.

Since the functions $N_{w}$ are norms, the adelic Lipschitz system $\mathcal{N}$ satisfies the hypothesis of Theorem 6.1. As our choice of $\mathcal{R}, S_{C}$ and $T$ in Theorem 6.1 will depend only on $k, n$ and $\theta$, we obtain

$$
N_{\mathcal{N}}\left(\mathbb{P}^{n}(k), X\right)=\omega_{k}^{-1}(n+1)^{r+s-1} R_{k} V_{\mathcal{N}^{\prime}} g_{k}^{\mathcal{N}} X^{d(n+1)}+O\left(X^{d(n+1)-1} \mathfrak{L}\right),
$$

where $\mathfrak{L}:=\log (X+1)$ if $(n, d)=(1,1)$ and $\mathfrak{L}:=1$ otherwise. The implicit constant in the error term depends only on $k, \theta$, and $n$.

We notice that

$$
V_{\mathcal{N}^{\prime}}=\left(2^{r} \pi^{s}\right)^{n+1} V(\theta, k, n),
$$

with $V(\theta, k, n)$ as in (1.3). To prove the theorem, we need to compute $g_{k}^{\mathcal{N}}$. First we choose the sets $\mathcal{R}, S_{C}$ and $T$. Denote

$$
D:={ }^{\mathfrak{d}}\left(\theta \mathcal{O}_{K}\right)
$$

For $\mathcal{R}$ we choose any system of integral representatives for the class group $\mathrm{Cl}_{k}$ with

$$
(C, D)=\mathcal{O}_{k} \text { for all } C \in \mathcal{R} \text {. }
$$

We will see in Lemma 8.2, (i), that

$$
S_{C}:=\left\{{ }^{\mathfrak{u}} C\left(\theta \mathcal{O}_{K},{ }^{\mathfrak{u}} B\right): B \unlhd \mathcal{O}_{k}, B \mid D\right\}
$$

is a valid choice for $S_{C}$. For $T$, we take the finite set

$$
T:=\bigcup_{C \in \mathcal{R}} \bigcup_{\mathfrak{D} \in S_{C}} T_{C, \mathfrak{D}} \cup\left\{\mathfrak{A} \unlhd \mathcal{O}_{K}: \mathfrak{A} \mid \theta \mathcal{O}_{K}\right\} .
$$




\section{Lemma 8.2.}

(i) Let $\boldsymbol{\omega} \in k^{n+1}$ with $\mathcal{O}_{k}(\boldsymbol{\omega})=C$. Then $i_{\mathcal{N}}(\boldsymbol{\omega}) \in S_{C}$.

(ii) Let $\mathfrak{A}$ be an ideal of $\mathcal{O}_{K}$ and $B$ an ideal of $\mathcal{O}_{k}$. Then ${ }^{\mathfrak{d}}\left(\mathfrak{A},{ }^{\mathfrak{u}} B\right)=\left({ }^{\mathfrak{d}} \mathfrak{A}, B\right)$.

(iii) Let $B$ be an ideal of $\mathcal{O}_{k}$ with $B \mid D$. Then ${ }^{\mathfrak{d}}\left(\theta \mathcal{O}_{K},{ }^{\mathfrak{u}} B\right)=B$.

Proof. (i): We have $\omega_{0} \mathcal{O}_{K}+\cdots+\omega_{n} \mathcal{O}_{K}={ }^{\mathfrak{u}} \mathcal{O}_{k}(\omega)={ }^{\mathfrak{u}} C$, so

$$
\begin{aligned}
i_{\mathcal{N}}(\boldsymbol{\omega}) & ={ }^{\mathfrak{u}} C\left(\omega_{0}\left({ }^{\mathfrak{u}} C\right)^{-1}+\theta\left(\omega_{1}\left({ }^{\mathfrak{u}} C\right)^{-1}+\cdots+\omega_{n}\left({ }^{\mathfrak{u}} C\right)^{-1}\right)\right) \\
& ={ }^{\mathfrak{u}} C\left(\omega_{0}\left({ }^{\mathfrak{u}} C\right)^{-1}, \theta \mathcal{O}_{K}\right) .
\end{aligned}
$$

Moreover, since $\theta \mathcal{O}_{K} \mid{ }^{\mathfrak{u}} D$, we obtain

$$
\left(\omega_{0}\left({ }^{\mathfrak{u}} C\right)^{-1}, \theta \mathcal{O}_{K}\right)=\left(\omega_{0}\left({ }^{\mathfrak{u}} C\right)^{-1},{ }^{\mathfrak{u}} D, \theta \mathcal{O}_{K}\right)=\left(\theta \mathcal{O}_{K},{ }^{\mathfrak{u}} B\right),
$$

for $B:=\left(\omega_{0} C^{-1}, D\right) \mid D$. Then

(ii): Let $P$ be a prime ideal of $\mathcal{O}_{k}$ and ${ }^{\mathfrak{u}} P=\prod_{\mathfrak{P}} \mathfrak{P}^{e \mathfrak{P}}$ its factorization in $\mathcal{O}_{K}$.

$$
\begin{aligned}
v_{P}\left({ }^{\mathfrak{d}}\left(\mathfrak{A},{ }^{\mathfrak{u}} B\right)\right) & =\max _{\mathfrak{P}}\left\{\left\lceil\min \left\{v_{\mathfrak{P}}(\mathfrak{A}), v_{\mathfrak{P}}\left({ }^{\mathfrak{u}} B\right)\right\} / e_{\mathfrak{P}}\right\rceil\right\} \\
& =\max _{\mathfrak{P}}\left\{\min \left\{\left\lceil v_{\mathfrak{P}}(\mathfrak{A}) / e_{\mathfrak{P}}\right\rceil, v_{P}(B)\right\}\right\} \\
& =\min \left\{\max _{\mathfrak{P}}\left\{\left\lceil v_{\mathfrak{P}}(\mathfrak{A}) / e_{\mathfrak{P}}\right\rceil\right\}, v_{P}(B)\right\}=v_{P}\left(\left({ }^{\mathfrak{d}} \mathfrak{A}, B\right)\right) .
\end{aligned}
$$

(iii): By (ii), we have ${ }^{\mathfrak{d}}\left(\theta \mathcal{O}_{K},{ }^{\mathfrak{u}} B\right)=(D, B)=B$.

The first step in our computation of $g_{k}^{\mathcal{N}}$ is to evaluate the determinant of the lattice $\Lambda(\mathfrak{A D}, C E)=\Lambda(\mathfrak{A D}) \cap \sigma\left((C E)^{n+1}\right)$.

Lemma 8.3. Let $\mathfrak{A}, B$ be nonzero ideals of $\mathcal{O}_{K}$ and $\mathcal{O}_{k}$, respectively. Then

$$
\operatorname{det} \Lambda(\mathfrak{A}, B)=\left(2^{-s} \sqrt{\left|\Delta_{k}\right|}\right)^{n+1} \cdot \mathfrak{N}_{k}\left({ }^{\mathfrak{d}} \mathfrak{A} \cap B\right) \cdot \mathfrak{N}_{k}\left({ }^{\mathfrak{d}}\left(\mathfrak{A}\left(\theta \mathcal{O}_{K}, \mathfrak{A}\right)^{-1}\right) \cap B\right)^{n}
$$

Proof. Let $\boldsymbol{\omega}=\left(\omega_{0}, \ldots, \omega_{n}\right) \in k^{n}$. Clearly, $\sigma \boldsymbol{\omega} \in \Lambda(\mathfrak{A}, B)$ if and only if $\omega_{i} \in B$ for all $0 \leq i \leq n, \omega_{0} \in \mathfrak{A}$, and $\theta \omega_{i} \in \mathfrak{A}$ for all $1 \leq i \leq n$. For $\omega_{i} \in \mathcal{O}_{k}$, we have

$$
\theta \omega_{i} \in \mathfrak{A} \quad \text { if and only if } \quad \mathfrak{A}\left(\theta \mathcal{O}_{K}, \mathfrak{A}\right)^{-1} \mid \omega_{i} \mathcal{O}_{K}
$$

Therefore, we obtain

$$
\Lambda(\mathfrak{A}, B)=\sigma\left(\left({ }^{\mathfrak{d}} \mathfrak{A} \cap B\right) \times\left({ }^{\mathfrak{d}}\left(\mathfrak{A}\left(\theta \mathcal{O}_{K}, \mathfrak{A}\right)^{-1}\right) \cap B\right)^{n}\right)
$$


Let $\mathfrak{A} \in T$ and let $B$ be an ideal of $\mathcal{O}_{k}$ with $B \mid D$. To facilitate further notation, we define ideals $A$ and $A_{1}$ of $\mathcal{O}_{k}$ by

$$
\begin{aligned}
A=A(\mathfrak{A}, B) & :={ }^{\mathfrak{d}}\left(\mathfrak{A}\left(\theta \mathcal{O}_{K},{ }^{\mathfrak{u}} B\right)\right) \quad \text { and } \\
A_{1}=A_{1}(\mathfrak{A}, B) & :={ }^{\mathfrak{d}}\left(\mathfrak{A}\left(\theta \mathcal{O}_{K},{ }^{\mathfrak{u}} B\right)\left(\theta \mathcal{O}_{K}, \mathfrak{A}^{\mathfrak{u}} B\right)^{-1}\right) \mid A .
\end{aligned}
$$

For any $\mathfrak{D}={ }^{\mathfrak{u}} C\left(\theta \mathcal{O}_{K},{ }^{\mathfrak{u}} B\right) \in S_{C}$ and for any nonzero ideal $E$ of $\mathcal{O}_{k}$ we have

$$
\mathfrak{N}_{k}\left({ }^{\mathfrak{d}}(\mathfrak{A D}) \cap C E\right)=\mathfrak{N}_{k} C \cdot \mathfrak{N}_{k}(A \cap E) .
$$

Clearly, we have $\left(\theta \mathcal{O}_{K}, \mathfrak{A}\left(\theta \mathcal{O}_{K},{ }^{\mathfrak{u}} B\right)\right)=\left(\theta \mathcal{O}_{K}, \mathfrak{A}^{\mathfrak{u}} B\right)$. Furthermore, by our choice of $\mathcal{R}$ with (8.5), we have $\left({ }^{\mathfrak{u}} C, \theta \mathcal{O}_{K}\right)=\mathcal{O}_{K}$. Therefore, we obtain

$$
\mathfrak{N}_{k}\left(\mathfrak{d}\left((\mathfrak{A D})\left(\theta \mathcal{O}_{K}, \mathfrak{A D}\right)^{-1}\right) \cap C E\right)=\mathfrak{N}_{k} C \cdot \mathfrak{N}_{k}\left(A_{1} \cap E\right) .
$$

Moreover, we have

$$
\mathfrak{N}_{K} \mathfrak{D}^{(n+1) /[K: k]}=\mathfrak{N}_{k} C^{n+1} \cdot \mathfrak{N}_{K}\left(\theta \mathcal{O}_{K},{ }^{\mathfrak{u}} B\right)^{(n+1) /[K: k]} .
$$

Lemma 8.4. Let $B$ be an ideal of $\mathcal{O}_{k}$ with $B \mid D$, let $\mathfrak{D}={ }^{\mathfrak{u}} C\left(\theta \mathcal{O}_{K},{ }^{\mathfrak{u}} B\right) \in S_{C}$, let $\mathfrak{A} \in T$, and let $E$ be a nonzero ideal of $\mathcal{O}_{k}$. Then

$$
\frac{\mathfrak{N}_{K} \mathfrak{D}^{\frac{n+1}{[K: k]}}}{\operatorname{det} \Lambda(\mathfrak{A D}, C E)}=\left(2^{-s} \sqrt{\left|\Delta_{k}\right|}\right)^{-(n+1)} \cdot \frac{\mathfrak{N}_{K}\left(\theta \mathcal{O}_{K},{ }^{\mathfrak{u}} B\right)^{\frac{n+1}{[K: k]}}}{\mathfrak{N}_{k}(A \cap E) \cdot \mathfrak{N}_{k}\left(A_{1} \cap E\right)^{n}} .
$$

Proof. We apply Lemma 8.3 and use (8.10), (8.11), and (8.12).

Lemma 8.5. We have

$$
g_{k}^{\mathcal{N}}=c_{0} \sum_{B \mid D} \mathfrak{N}_{K}\left(\theta \mathcal{O}_{K},{ }^{\mathfrak{u}} B\right)^{\frac{n+1}{[K: k]}} \sum_{\mathfrak{A} \in T} \mu_{K}(\mathfrak{A}) \sum_{E \unlhd \mathcal{O}_{k}} \frac{\mu_{k}(E)}{\mathfrak{N}_{k}(A \cap E) \cdot \mathfrak{N}_{k}\left(A_{1} \cap E\right)^{n}},
$$

where $A=A(\mathfrak{A}, B), A_{1}=A_{1}(\mathfrak{A}, B)$, and $c_{0}:=h_{k} 2^{s(n+1)}\left(\sqrt{\left|\Delta_{k}\right|}\right)^{-(n+1)}$ and $E$ runs over all nonzero ideals of $\mathcal{O}_{k}$.

Proof. Recall the definition of $g_{k}^{\mathcal{N}}$ in (6.6). The expression on the right-hand side in Lemma 8.4 does not depend on $C$. With (8.6), a simple computation proves the lemma.

The inner sum over $E$ in Lemma 8.5 can be handled by the following lemma.

Lemma 8.6. Let $J_{1} \mid J$ be nonzero ideals of $\mathcal{O}_{k}$ and let

$$
\xi:=\sum_{E \unlhd \mathcal{O}_{k}} \frac{\mu_{k}(E)}{\mathfrak{N}_{k}(J \cap E) \cdot \mathfrak{N}_{k}\left(J_{1} \cap E\right)^{n}} .
$$

If $J_{1} \neq \mathcal{O}_{k}$ then $\xi=0$. If $J_{1}=\mathcal{O}_{k}$ then

$$
\xi=\frac{1}{\zeta_{k}(n+1) \mathfrak{N}_{k}(J)} \prod_{P \mid J} \frac{\mathfrak{N}_{k} P^{n+1}-\mathfrak{N}_{k} P}{\mathfrak{N}_{k} P^{n+1}-1} .
$$


Proof. Let $f(E):=\mu_{k}(E) \cdot \mathfrak{N}_{k}(J, E) \cdot \mathfrak{N}_{k}\left(J_{1}, E\right)^{n}$. Then $f$ is multiplicative and

$$
\xi=\frac{1}{\mathfrak{N}_{k}\left(J J_{1}^{n}\right)} \sum_{E \unlhd \mathcal{O}_{k}} \frac{f(E)}{\mathfrak{N}_{k} E^{n+1}} .
$$

Clearly, this Dirichlet series converges absolutely for all $n>0$. Let us compute its Euler product expansion. For any prime ideal $P$ of $\mathcal{O}_{k}$, we have $f\left(P^{e}\right)=0$ if $e \geq 2$. Moreover, $f\left(\mathcal{O}_{k}\right)=1$ and

$$
f(P)= \begin{cases}-\mathfrak{N}_{k} P^{n+1} & \text { if } P \mid J_{1}, \\ -\mathfrak{N}_{k} P & \text { if } P \mid J \text { and } P \nmid J_{1}, \\ -1 & \text { if } P \nmid J .\end{cases}
$$

We obtain the formal expansion

$$
\sum_{E \unlhd \mathcal{O}_{k}} \frac{f(E)}{\mathfrak{N}_{k} E^{s}}=\prod_{P \mid J_{1}}\left(1-\frac{\mathfrak{N}_{k} P^{n+1}}{\mathfrak{N}_{k} P^{s}}\right) \prod_{\substack{P \mid J \\ P \nmid J_{1}}}\left(1-\frac{\mathfrak{N}_{k} P}{\mathfrak{N}_{k} P^{s}}\right) \prod_{P \nmid J}\left(1-\frac{1}{\mathfrak{N}_{k} P^{s}}\right) .
$$

Since the infinite product $\prod_{P \nmid J}\left(1-\mathfrak{N}_{k} P^{-s}\right)$ converges absolutely for $s>1$, we obtain $\xi=0$ whenever $J_{1} \neq \mathcal{O}_{k}$. If $J_{1}=\mathcal{O}_{k}$ and $s=n+1$, the expression simplifies to

$$
\sum_{E \unlhd \mathcal{O}_{k}} \frac{f(E)}{\mathfrak{N}_{k} E^{n+1}}=\frac{1}{\zeta_{k}(n+1)} \prod_{P \mid J} \frac{\mathfrak{N}_{k} P^{n+1}-\mathfrak{N}_{k} P}{\mathfrak{N}_{k} P^{n+1}-1} .
$$

Recall the definition of $A$ and $A_{1}$ from (8.8) and (8.9). We have $A_{1}=\mathcal{O}_{k}$ if and only if $\mathfrak{A}\left(\theta \mathcal{O}_{K},{ }^{\mathfrak{u}} B\right)=\left(\theta \mathcal{O}_{K}, \mathfrak{A}^{\mathfrak{u}} B\right)$, which is equivalent to $\mathfrak{A}\left(\theta \mathcal{O}_{K},{ }^{\mathfrak{u}} B\right) \mid \theta \mathcal{O}_{K}$, or

$$
\mathfrak{A} \mid \theta \mathcal{O}_{K}\left(\theta \mathcal{O}_{K},{ }^{\mathfrak{u}} B\right)^{-1} \text {. }
$$

Recall that, by (8.7), the set $T$ contains all ideals $\mathfrak{A}$ of $\mathcal{O}_{K}$ with $\mathfrak{A} \mid \theta \mathcal{O}_{K}$. Also, for every $\mathfrak{A}$ with (8.13), we have $A={ }^{\mathfrak{d}}\left(\mathfrak{A}\left(\theta \mathcal{O}_{K},{ }^{\mathfrak{u}} B\right)\right) \mid D$. We obtain

$$
g_{k}^{\mathcal{N}}=c_{1} \sum_{B \mid D} \mathfrak{N}_{K}\left(\theta \mathcal{O}_{K},{ }^{\mathfrak{u}} B\right)^{\frac{n+1}{[K: k]}} \sum_{A \mid D} \frac{1}{\mathfrak{N}_{k} A} \prod_{P \mid A} \frac{\mathfrak{N}_{k} P^{n+1}-\mathfrak{N}_{k} P}{\mathfrak{N}_{k} P^{n+1}-1} s_{0}(A, B),
$$

where $c_{1}:=\zeta_{k}(n+1)^{-1} c_{0}=h_{k} 2^{s(n+1)} \zeta_{k}(n+1)^{-1}\left(\sqrt{\left|\Delta_{k}\right|}\right)^{-(n+1)}$ and

$$
s_{0}(A, B):=\sum_{\substack{\mathfrak{A} \text { with }(8.13) \\ \mathfrak{0}\left(\mathfrak{A}\left(\theta \mathcal{O}_{K},{ }^{u} B\right)\right)=A}} \mu_{K}(\mathfrak{A}) .
$$

If $s_{0}(A, B)$ is not zero then there is at least one $\mathfrak{A}$ with

$$
A={ }^{\mathfrak{d}}\left(\mathfrak{A}\left(\theta \mathcal{O}_{K},{ }^{\mathfrak{u}} B\right)\right) \subseteq{ }^{\mathfrak{d}}\left(\theta \mathcal{O}_{K},{ }^{\mathfrak{u}} B\right)=B .
$$


For the last equality, we used Lemma 8.2 , (iii). We replace $A$ by $B^{-1} A$ to obtain

$$
g_{k}^{\mathcal{N}}=c_{1} \sum_{B \mid D} \frac{\mathfrak{N}_{K}\left(\theta \mathcal{O}_{K},{ }^{\mathfrak{u}} B\right)^{\frac{n+1}{[K: k]}}}{\mathfrak{N}_{k} B} \sum_{A \mid B^{-1} D} \frac{1}{\mathfrak{N}_{k} A} \prod_{P \mid A B} \frac{\mathfrak{N}_{k} P^{n+1}-\mathfrak{N}_{k} P}{\mathfrak{N}_{k} P^{n+1}-1} s(A, B),
$$

where

$$
s(A, B):=\sum_{\substack{\mathfrak{A} \text { with }(8.13) \\ \mathfrak{d}\left(\mathfrak{A}\left(\theta \mathcal{O}_{K},{ }^{\mathfrak{u}} B\right)\right)=A B}} \mu_{K}(\mathfrak{A}) .
$$

Lemma 8.7. Let $\mathfrak{J}, \mathfrak{K}$ be nonzero ideals of $\mathcal{O}_{K}$ and $J$ a nonzero ideal of $\mathcal{O}_{k}$. Then ${ }^{\mathfrak{d}}(\mathfrak{J} \mathfrak{K})=J^{\mathfrak{d} \mathfrak{K}}$ if and only if

$$
\left.\mathfrak{J}\right|^{\mathfrak{u}} J\left({ }^{\mathfrak{u} \mathfrak{K}} \mathfrak{K}\right) \mathfrak{K}^{-1} \text { and } \mathfrak{J} \nmid \mathfrak{u}\left(P^{-1} J\right)\left({ }^{\mathfrak{u} \mathfrak{d}} \mathfrak{K}\right) \mathfrak{K}^{-1} \text { for all prime ideals } P \mid J \text {. }
$$

Proof. Clearly,

$$
\left.\left.\mathfrak{J}\right|^{\mathfrak{u}} J\left({ }^{\mathfrak{u} \mathfrak{d}} \mathfrak{K}\right) \mathfrak{K}^{-1} \Longleftrightarrow \mathfrak{J} \mathfrak{K}\right|^{\mathfrak{u}}\left(J^{\mathfrak{d}} \mathfrak{K}\right) \Longleftrightarrow{ }^{\mathfrak{d}}(\mathfrak{J} \mathfrak{K}) \mid J^{\mathfrak{d}} \mathfrak{K}
$$

and

$$
\mathfrak{J} \nmid^{\mathfrak{u}}\left(P^{-1} J\right)\left({ }^{\mathfrak{u} \mathfrak{d}} \mathfrak{K}\right) \mathfrak{K}^{-1} \Longleftrightarrow \mathfrak{J} \mathfrak{K} \nmid^{\mathfrak{u}}\left(P^{-1} J^{\mathfrak{d}} \mathfrak{K}\right) \Longleftrightarrow{ }^{\mathfrak{d}}(\mathfrak{J} \mathfrak{K}) \nmid\left(P^{-1} J\right)^{\mathfrak{d}} \mathfrak{K} .
$$

Lemma 8.8. If $A \mid B^{-1} D$ then $s(A, B)=\mu_{k}(A)$.

Proof. By Lemma 8.2, (iii), we have ${ }^{\mathfrak{d}}\left(\theta \mathcal{O}_{K},{ }^{\mathfrak{u}} B\right)=B$. By the previous lemma, $\mathfrak{d}\left(\mathfrak{A}\left(\theta \mathcal{O}_{K},{ }^{\mathfrak{u}} B\right)\right)=A B$ is equivalent to

$$
\mathfrak{A} \mid{ }^{\mathfrak{u}} A^{\mathfrak{u}} B\left(\theta \mathcal{O}_{K},{ }^{\mathfrak{u}} B\right)^{-1} \text { and } \mathfrak{A} \nmid{ }^{\mathfrak{u}}\left(P^{-1} A\right){ }^{\mathfrak{u}} B\left(\theta \mathcal{O}_{K},{ }^{\mathfrak{u}} B\right)^{-1} \text { for all } P \mid A
$$

Clearly, conditions (8.13) and (8.15) imply

$$
\mathfrak{A} \mid\left(\theta \mathcal{O}_{K}\left(\theta \mathcal{O}_{K},{ }^{\mathfrak{u}} B\right)^{-1},{ }^{\mathfrak{u}} A^{\mathfrak{u}} B\left(\theta \mathcal{O}_{K},{ }^{\mathfrak{u}} B\right)^{-1}\right)=\left(\theta \mathcal{O}_{K}\left(\theta \mathcal{O}_{K},{ }^{\mathfrak{u}} B\right)^{-1},{ }^{\mathfrak{u}} A\right)
$$

and

$$
\mathfrak{A} \nmid^{\mathfrak{u}}\left(P^{-1} A\right) \text { for all prime ideals } P \mid A .
$$

In fact, (8.13) and (8.15) are equivalent to (8.16) and (8.17). Indeed, (8.16) immediately implies (8.13) and the first part of (8.15). For the second part of (8.15), we use that every $\mathfrak{A} \mid \theta \mathcal{O}_{K}\left(\theta \mathcal{O}_{K},{ }^{\mathfrak{u}} B\right)^{-1}$ satisfies $\left(\mathfrak{A},{ }^{\mathfrak{u}} B\left(\theta \mathcal{O}_{K},{ }^{\mathfrak{u}} B\right)^{-1}\right)=\mathcal{O}_{K}$. Thus,

$$
s(A, B)=\sum_{\substack{\mathfrak{A} \unlhd \mathcal{O}_{K} \\(8.13) \text { and }(8.15)}} \mu_{K}(\mathfrak{A})=\sum_{\substack{\mathfrak{A} \unlhd \mathcal{O}_{K} \\(8.16) \text { and }(8.17)}} \mu_{K}(\mathfrak{A}) .
$$


By inclusion-exclusion for (8.17), we obtain

$$
s(A, B)=\sum_{F \mid A} \mu_{k}(F) \sum_{\mathfrak{A} \mid\left(\theta \mathcal{O}_{K}\left(\theta \mathcal{O}_{K},{ }^{\mathfrak{u}} B\right)^{-1},{ }^{\mathfrak{u}}\left(F^{-1} A\right)\right)} \mu_{K}(\mathfrak{A}) .
$$

The last sum is 1 if $F=A$. Moreover,

$$
F^{-1} A\left|B^{-1} D={ }^{\mathfrak{d}}\left(\theta \mathcal{O}_{K}\right)\left({ }^{\mathfrak{o}}\left(\theta \mathcal{O}_{K},{ }^{\mathfrak{u}} B\right)\right)^{-1}\right|{ }^{\mathfrak{o}}\left(\theta \mathcal{O}_{K}\left(\theta \mathcal{O}_{K},{ }^{\mathfrak{u}} B\right)^{-1}\right),
$$

so $F \neq A$ implies that

$$
\left(\theta \mathcal{O}_{K}\left(\theta \mathcal{O}_{K},{ }^{\mathfrak{u}} B\right)^{-1},{ }^{\mathfrak{u}}\left(F^{-1} A\right)\right) \neq \mathcal{O}_{K} .
$$

This shows that the last sum is 0 whenever $F \neq A$.

We obtain

$$
g_{k}^{\mathcal{N}}=c_{1} \sum_{B \mid D} \frac{\mathfrak{N}_{K}\left(\theta \mathcal{O}_{K},{ }^{\mathfrak{u}} B\right)^{(n+1) /[K: k]}}{\mathfrak{N}_{k} B} \sum_{A \mid B^{-1} D} \frac{\mu_{k}(A)}{\mathfrak{N}_{k} A} \prod_{P \mid A B} \frac{\mathfrak{N}_{k} P^{n+1}-\mathfrak{N}_{k} P}{\mathfrak{N}_{k} P^{n+1}-1},
$$

and Theorem 1.4 follows by substituting this and (8.4) in (8.3).

\section{Proof of Theorem 1.3}

In this section we will use not only Landau's $O$-notation but also Vinogradov's symbol $\ll$. All implied constants depend solely on $k$. As we will encounter expressions like $\log \log X$ we assume throughout the entire section that $X \geq 3$. Our main task will be to prove the following proposition.

Proposition 9.1. Suppose $p \in \mathbf{P}_{k}$. Then, as $X \geq 3$ tends to infinity, we have

$$
N\left(\sqrt{p} k^{*}, X\right)=\frac{2 p^{d / 2}}{p^{d}+1} S_{k}(1) X^{2 d}+O\left(\frac{X^{2 d-1}}{p^{(d-1) / 2}}+X^{d} \log X+X^{d} \log p\right) .
$$

We choose the adelic Lipschitz system $\mathcal{N}$ (of dimension 1) on $K:=k(\sqrt{p})$, defined by

$$
N_{w}\left(\left(z_{0}, z_{1}\right)\right):=\max \left\{\left|z_{0}\right|_{w},|\sqrt{p}|_{w}\left|z_{1}\right|_{w}\right\}
$$

for any place $w$ of $K$. Recall the definition of $C_{\mathcal{N}}^{\text {fin }}$ and $C_{\mathcal{N}}^{\text {inf }}$ from (5.5) and (5.6), and note that we can take

$$
C_{\mathcal{N}}^{\mathrm{fin}}=C_{\mathcal{N}}^{\mathrm{inf}}=\sqrt{p}
$$

The adelic Lipschitz system $\mathcal{N}$ on $K$ leads to an adelic Lipschitz system $\mathcal{N}^{\prime}$ on $k$ as in Section 6. Note that for any Archimedean $v$ from $k$ and $N_{v}$ from $\mathcal{N}^{\prime}$ we have $N_{v}\left(\left(z_{0}, z_{1}\right)\right)=\max \left\{\left|z_{0}\right|_{v}, \sqrt{p}\left|z_{1}\right|_{v}\right\}$. Thus we can also take

$$
C_{\mathcal{N}^{\prime}}^{\inf }=\sqrt{p} .
$$


Lemma 9.2. We have

$$
N\left(\sqrt{p} k^{*}, X\right)=N_{\mathcal{N}}\left(\mathbb{P}^{1}(k) ; X\right)-2 .
$$

Proof. The map $\alpha \mapsto(1: \alpha)$ is a one-to-one correspondence between $k^{*}$ and $\mathbb{P}^{1}(k) \backslash\{(0: 1),(1: 0)\}$ Moreover, $H(\sqrt{p} \alpha)=H_{\mathcal{N}}((1: \alpha))$. Hence there is a oneto-one correspondence between $\left\{\alpha \in k^{*}: H(\sqrt{p} \alpha) \leq X\right\}$ and $\left\{P \in \mathbb{P}^{1}(k) \backslash\{(0\right.$ : $\left.1),(1: 0)\}: H_{\mathcal{N}}(P) \leq X\right\}$. As $H_{\mathcal{N}}((0: 1))=H_{\mathcal{N}}((1: 0))=1$ the claim follows.

We can now basically follow the proof of Theorem 6.1 using our specific adelic Lipschitz system. However, to get the good error terms regarding $p$ an additional idea is required. We will use the same notation as in Sections 6 and 7. In particular, recall the definition of the set $S_{F}(t)$ introduced in (7.2). As in (8.5), we choose a system $\mathcal{R}$ of integral representatives for $\mathrm{Cl}_{k}$ such that $\left(C, p \mathcal{O}_{k}\right)=\mathcal{O}_{k}$ for all $C \in \mathcal{R}$.

Lemma 9.3. We can choose $S_{C}:=\left\{{ }^{\mathfrak{u}} C, \sqrt{p}^{\mathfrak{u}} C\right\}$.

Proof. As in (8.1) we have $i_{\mathcal{N}}(\boldsymbol{\omega})=\omega_{0} \mathcal{O}_{K}+\sqrt{p} \omega_{1} \mathcal{O}_{K}$. So if $\mathcal{O}_{k}(\boldsymbol{\omega})=C$ we get $\sqrt{p}^{\mathfrak{u}} C \subseteq i_{\mathcal{N}}(\omega) \subseteq{ }^{\mathfrak{u}} C$. As $\sqrt{p} \mathcal{O}_{K}$ is a prime ideal this proves the lemma.

With this choice of the sets $S_{C}$ we directly verify that $\Gamma$ from (7.13) can be chosen to be

$$
\Gamma:=p^{-d / 2}
$$

From now on $C$ is always in $\mathcal{R}, \mathfrak{D}$ is always in $S_{C}$, and $\mathfrak{A}$ will always be in $T$.

Lemma 9.4. We can choose $T$ such that $|T| \leq 2$.

Proof. Recall that we may choose $T=\cup_{C \in \mathcal{R}} \cup_{\mathfrak{D} \in S_{C}} T_{C, \mathfrak{D}}$. By definition we have

$$
\begin{aligned}
T_{C, \mathfrak{D}} & =\left\{\mathfrak{B} \unlhd \mathcal{O}_{K}: \Lambda_{C}(\mathfrak{D} \mathfrak{B}) \neq \emptyset\right\} \\
& =\left\{\mathfrak{B} \unlhd \mathcal{O}_{K}: \Lambda_{C}^{*}(\mathfrak{E D B}) \neq \emptyset \text { for some } \mathfrak{E} \unlhd \mathcal{O}_{K}\right\} \\
& \subseteq\left\{\mathfrak{B} \unlhd \mathcal{O}_{K}: \mathfrak{E} \mathfrak{D} \mathfrak{B} \in S_{C} \text { for some } \mathfrak{E} \unlhd \mathcal{O}_{K}\right\} .
\end{aligned}
$$

Now using that $S_{C}=\left\{{ }^{\mathfrak{u}} C, \sqrt{p^{\mathfrak{u}} C}\right.$ and that $\sqrt{p} \mathcal{O}_{K}$ is a prime ideal we see that $T_{C, \mathfrak{D}} \subseteq\left\{\mathcal{O}_{K}, \sqrt{p} \mathcal{O}_{K}\right\}$ for any $\mathfrak{D} \in S_{C}$. Thus $|T|=\left|\cup_{C \in \mathcal{R}} \cup_{\mathfrak{D} \in S_{C}} T_{C, \mathfrak{D}}\right| \leq 2$.

Lemma 9.5. Let $\sigma$ be as in (6.3). We have

$$
\Lambda(\mathfrak{A D}, C E) \subseteq \sigma(C E) \times \sigma(C E) .
$$

Moreover, if $\mathfrak{D}=\sqrt{p}^{\mathfrak{u}} C$ then we have

$$
\Lambda(\mathfrak{A D}, C E) \subseteq \sigma\left(C E p\left(C E, p \mathcal{O}_{k}\right)^{-1}\right) \times \sigma(C E)
$$


Proof. The first assertion is clear from the definition. For the second assertion we could use the last equality in the proof of Lemma 8.3, but we prefer to give a direct argument here. Note that $\sigma \boldsymbol{\omega} \in \Lambda(\mathfrak{A D})$ implies $\mathfrak{D} \mid i_{\mathcal{N}}(\boldsymbol{\omega})=\left(\omega_{0} \mathcal{O}_{K}, \sqrt{p} \omega_{1} \mathcal{O}_{K}\right)$. As $\mathfrak{D}=\sqrt{p}^{\mathfrak{u}} C$ we conclude $\sqrt{p} \mathcal{O}_{K} \mid \omega_{0} \mathcal{O}_{K}$, and thus $p \mathcal{O}_{k} \mid \omega_{0} \mathcal{O}_{k}$. Therefore $\omega_{0} \in C E \cap p \mathcal{O}_{k}$. This proves the second assertion.

Next we use a trick, simpler but reminiscent of those used in [20, Section 6]. To this end we introduce a linear automorphism $\Phi$ of determinant 1 on $\left(\mathbb{R}^{r} \times \mathbb{C}^{s}\right)^{2}$ by

$$
\Phi\left(\mathbf{z}_{0}, \mathbf{z}_{1}\right):=\left(p^{-1 / 4} \mathbf{z}_{0}, p^{1 / 4} \mathbf{z}_{1}\right)
$$

Lemma 9.6. Write $\Lambda:=\Lambda(\mathfrak{A D}, C E)$. If $\mathfrak{D}={ }^{\mathfrak{u}} C$ then we have

$$
\begin{aligned}
\lambda_{1}(\Phi \Lambda) & \geq p^{-1 / 4} \mathfrak{N}_{k}(C E)^{1 / d} \\
\lambda_{d+1}(\Phi \Lambda) & \geq p^{1 / 4} \mathfrak{N}_{k}(C E)^{1 / d}
\end{aligned}
$$

If $\mathfrak{D}=\sqrt{p}^{\mathfrak{u}} C$ then we have

$$
\begin{gathered}
\lambda_{1}(\Phi \Lambda) \geq \begin{cases}p^{-1 / 4} \mathfrak{N}_{k}(C E)^{1 / d} & \text { if } p \mathcal{O}_{k} \mid E, \\
p^{1 / 4} \mathfrak{N}_{k}(C E)^{1 / d} & \text { if } p \mathcal{O}_{k} \nmid E .\end{cases} \\
\lambda_{d+1}(\Phi \Lambda) \geq \begin{cases}p^{1 / 4} \mathfrak{N}_{k}(C E)^{1 / d} & \text { if } p \mathcal{O}_{k} \mid E, \\
p^{3 / 4} \mathfrak{N}_{k}(C E)^{1 / d} & \text { if } p \mathcal{O}_{k} \nmid E .\end{cases}
\end{gathered}
$$

Proof. By Lemma 9.5 we have $\Phi \Lambda \subseteq \Lambda_{1} \times \Lambda_{2}$, where $\Lambda_{2}:=p^{1 / 4} \sigma(C E)$ and $\Lambda_{1}$ is $p^{-1 / 4} \sigma(C E)$ if $\mathfrak{D}={ }^{\mathfrak{u}} C$ and $p^{-1 / 4} \sigma\left(C E p\left(C E, p \mathcal{O}_{k}\right)^{-1}\right)$ if $\mathfrak{D}=\sqrt{p}^{\mathfrak{u}} C$. Recall the fact (already used in Lemma 7.4) that $\lambda_{1}(\sigma A) \geq \mathfrak{N}_{k} A^{1 / d}$ for any nonzero ideal $A$ of $k$. Using this and applying Lemma 4.5 the result follows from an easy computation.

Lemma 9.7. There exist constants $c_{1}=c_{1}(k)$ and $M=M(k)$ depending solely on $k$ such that, with $L=c_{1} p^{-1 / 4} t$, we have $\Phi S_{F}(t) \subseteq B_{0}(L)$ and the boundary $\partial \Phi S_{F}(t) \in \operatorname{Lip}(2 d, M, L)$.

Proof. The adelic Lipschitz system $\mathcal{N}$ on $K$ leads to an adelic Lipschitz system $\mathcal{N}^{\prime}$ on $k$ as in Section 6. The latter is used to define $S_{F}(t)$.

Now notice that applying $\Phi$ to $S_{F}(t)$ gives the same as defining $S_{F}(t)$ using the standard adelic Lipschitz system defined by $N_{v}\left(z_{0}, z_{1}\right)=\max \left\{\left|z_{0}\right|_{v},\left|z_{1}\right|_{v}\right\}$ for all $v$ and then homogeneously shrinking this set by the factor $p^{-1 / 4}$. The claims then follow immediately from Lemma 7.1, (7.9), and (7.8) applied to the standard adelic Lipschitz system. 
Lemma 9.8. Let $\mathcal{E}_{1}:=X^{d} / \mathfrak{N}_{k}(E)$, and let $\mathcal{E}_{2}:=X^{2 d-1} /\left(p^{(d-1) / 2} \mathfrak{N}_{k}(E)^{2-1 / d}\right)$. Then we have

$$
\begin{aligned}
\left|\Lambda(\mathfrak{A D}, C E) \cap S_{F}\left(X \mathfrak{N}_{K} \mathfrak{D}^{1 /(2 d)}\right)\right| & =\frac{\operatorname{Vol} S_{F}(1) \mathfrak{N}_{K} \mathfrak{D} X^{2 d}}{\operatorname{det} \Lambda(\mathfrak{A D}, C E)} \\
& +O\left(\left\{\begin{array}{ll}
\mathcal{E}_{1}+\mathcal{E}_{2} & \text { if } p \mathcal{O}_{k} \nmid E \\
p^{d / 2} \mathcal{E}_{1}+p^{d-1 / 2} \mathcal{E}_{2} & \text { if } p \mathcal{O}_{k} \mid E
\end{array}\right) .\right.
\end{aligned}
$$

Moreover, there is a constant $\gamma=\gamma(k) \geq 1$ depending only on $k$, such that $\left|\Lambda(\mathfrak{A D}, C E) \cap S_{F}\left(X \mathfrak{N}_{K} \mathfrak{D}^{1 /(2 d)}\right)\right|=0$ whenever $\mathfrak{N}_{k} E>(\gamma p X)^{d}$.

Proof. First note that

$\left|\Lambda(\mathfrak{A D}, C E) \cap S_{F}\left(X \mathfrak{N}_{K} \mathfrak{D}^{1 /(2 d)}\right)\right|=\left|\Phi \Lambda(\mathfrak{A D}, C E) \cap \Phi S_{F}\left(X \mathfrak{N}_{K} \mathfrak{D}^{1 /(2 d)}\right)\right|$.

Now we apply Lemma 4.2 with $a=d+1$ combined with Lemma 9.7 to conclude

$$
\begin{array}{r}
\left|\Phi \Lambda(\mathfrak{A D}, C E) \cap \Phi S_{F}\left(X \mathfrak{N}_{K} \mathfrak{D}^{1 /(2 d)}\right)\right|=\frac{\operatorname{Vol} S_{F}(1) \mathfrak{N}_{K} \mathfrak{D} X^{2 d}}{\operatorname{det} \Lambda(\mathfrak{A D}, C E)} \\
+O\left(\max \left\{\frac{p^{-d / 4} X^{d} \mathfrak{N}_{K} \mathfrak{D}^{1 / 2}}{\lambda_{1}(\Phi \Lambda)^{d}}, \frac{p^{-(2 d-1) / 4} X^{2 d-1} \mathfrak{N}_{K} \mathfrak{D}^{1-1 /(2 d)}}{\lambda_{1}(\Phi \Lambda)^{d} \lambda_{d+1}(\Phi \Lambda)^{d-1}}\right\}\right) .
\end{array}
$$

Finally, we use Lemma 9.6 to estimate $\lambda_{1}(\Phi \Lambda)$ and $\lambda_{d+1}(\Phi \Lambda)$, and the first claim follows from a simple computation. The second claim follows from Lemma 7.4 combined with (9.2) and (9.3).

We are now in the position to prove Proposition 9.1. In the introduction we already computed the main term, see (1.6). Proceeding exactly as in the proof of Theorem 6.1 in the case $(n, d)=(1,1)$, we obtain

$$
\begin{aligned}
N_{\mathcal{N}}\left(\mathbb{P}^{1}(k) ; X\right) & =\frac{2 p^{d / 2}}{p^{d}+1} S_{k}(1) X^{2 d} \\
& +O\left(\sum_{C \in \mathcal{R}} \sum_{\mathfrak{D} \in S_{C}} \sum_{\mathfrak{A} \in T} \sum_{\substack{E \unlhd \mathcal{O}_{k} \\
\mathfrak{N}_{k} E>(\gamma p X)^{d}}} \frac{\operatorname{Vol} \Phi S_{F}\left(X \mathfrak{N}_{K} \mathfrak{D}^{1 /(2 d)}\right)}{\operatorname{det} \Phi \Lambda(\mathfrak{A} \mathfrak{D}, C E)}\right) \\
& +O\left(\sum_{C \in \mathcal{R}} \sum_{\mathfrak{D} \in S_{C}} \sum_{\mathfrak{A} \in T} \sum_{\substack{E \unlhd \mathcal{O}_{k} \\
\mathfrak{N}_{k} E \leq(\gamma p X)^{d}}} \mathcal{E}_{1}+\mathcal{E}_{2}\right) \\
& +O\left(\sum_{C \in \mathcal{R}} \sum_{\mathfrak{D} \in S_{C}} \sum_{\mathfrak{A} \in T} \sum_{\substack{E \unlhd \mathcal{O}_{k} \\
\mathfrak{N}_{k} E \leq(\gamma p X)^{d} \\
p \mathcal{O}_{k} \mid E}} p^{d / 2} \mathcal{E}_{1}+p^{d-1 / 2} \mathcal{E}_{2}\right) .
\end{aligned}
$$


For the first error term we apply Minkowski's second theorem and Lemma 9.7 to get the upper bound

$$
\frac{\operatorname{Vol} \Phi S_{F}\left(X \mathfrak{N}_{K} \mathfrak{D}^{1 /(2 d)}\right)}{\operatorname{det} \Phi \Lambda(\mathfrak{A D}, C E)} \ll \frac{L^{2 d}}{\lambda_{1}(\Phi \Lambda)^{d} \lambda_{d+1}(\Phi \Lambda)^{d}},
$$

where $L \ll p^{-1 / 4} X \mathfrak{N}_{K} \mathfrak{D}^{1 /(2 d)}$. Summing the above over the finite sums can be handled by Lemmata 9.3 and 9.4. Now for the infinite sum over the ideals $E$, we apply Lemma 9.6, and a straightforward computation (using the dichotomy $P \mid E$, $P \nmid E$ ) yields the upper bound

$$
\ll \frac{X^{d}}{p^{3 d / 2}} .
$$

For the second error term we note that

$$
\sum_{\substack{E \unlhd \mathcal{O}_{k} \\ \mathfrak{N}_{k} E \leq(\gamma p X)^{d}}} \mathcal{E}_{1}=\sum_{\substack{E \unlhd \mathcal{O}_{k} \\ \mathfrak{N}_{k} E \leq(\gamma p X)^{d}}} \frac{X^{d}}{\mathfrak{N}_{k} E} \ll X^{d} \log \left((\gamma p X)^{d}\right) \ll X^{d} \log X+X^{d} \log p,
$$

and

$$
\sum_{\substack{E \unlhd \mathcal{O}_{k} \\ \mathfrak{N}_{k} E \leq(\gamma p X)^{d}}} \mathcal{E}_{2} \leq \frac{X^{2 d-1}}{p^{(d-1) / 2}} \sum_{E \unlhd \mathcal{O}_{k}} \mathfrak{N}_{k} E^{-2+1 / d} \ll \frac{X^{2 d-1}}{p^{(d-1) / 2}}
$$

Then we apply Lemmata 9.3 and 9.4 to conclude

$$
\sum_{C \in \mathcal{R}} \sum_{\mathfrak{D} \in S_{C}} \sum_{\mathfrak{A} \in T} \sum_{\substack{E \unlhd \mathcal{O}_{k} \\ \mathfrak{T}_{k} E \leq(\gamma p X)^{d}}} \mathcal{E}_{1}+\mathcal{E}_{2} \ll X^{d} \log X+X^{d} \log p+\frac{X^{2 d-1}}{p^{(d-1) / 2}} .
$$

Similar straightforward calculations yield

$$
\sum_{\substack{E \leq \mathcal{O}_{k} \\ \mathfrak{T}_{k} E \leq(\gamma p X)^{d} \\ p \mathcal{O}_{k} \mid E}} p^{d / 2} \mathcal{E}_{1} \ll \frac{X^{d}}{p^{d / 2}} \log X,
$$

and

$$
\sum_{\substack{E \leq \mathcal{O}_{k} \\ \mathfrak{F}_{k} E \leq(\gamma p X)^{d} \\ p \mathcal{O}_{k} \mid E}} p^{d-1 / 2} \mathcal{E}_{2} \ll \frac{X^{2 d-1}}{p^{3 d / 2-1}}
$$


Thus, applying again Lemmata 9.3 and 9.4 , we see that

$$
\sum_{C \in \mathcal{R}} \sum_{\mathfrak{D} \in S_{C}} \sum_{\mathfrak{A} \in T} \sum_{\substack{E \unlhd \mathcal{O}_{k} \\ \mathfrak{N}_{k} \in \leq(\gamma p X)^{d} \\ p \mathcal{O}_{k} \mid E}} p^{d / 2} \mathcal{E}_{1}+p^{d-1 / 2} \mathcal{E}_{2} \ll X^{d} \log X+\frac{X^{2 d-1}}{p^{(d-1) / 2}} .
$$

Combining these estimates and Lemma 9.2 completes the proof of Proposition 9.1.

We can now sum $N\left(\sqrt{p} k^{*}, X\right)$ over all $p \in \mathbf{P}_{k}$. The next lemma tells us that we can restrict the summation to $p \leq X^{2}$.

Lemma 9.9. For any $\alpha \in k^{*}$ and any $p \in \mathbf{P}_{k}$ we have $H(\sqrt{p} \alpha) \geq \sqrt{p}$.

Proof. Let $x \in K$ and let $\mathfrak{P}$ be the prime ideal $\sqrt{p} \mathcal{O}_{K}$. Then

$$
H(x) \geq \max \left\{1, \mathfrak{N}_{K} \mathfrak{P}\right\}^{-v_{\mathfrak{P}}\left(x \mathcal{O}_{K}\right) /(2 d)}=\max \left\{1, p^{d}\right\}^{-v_{\mathfrak{P}}\left(x \mathcal{O}_{K}\right) /(2 d)} .
$$

In particular, if $v_{\mathfrak{P}}\left(x \mathcal{O}_{K}\right)<0$ we get $H(x) \geq \sqrt{p}$. As $H(x)=H(1 / x)$ for any nonzero $x$ whatsoever, it suffices to show that the order of $\sqrt{p} \alpha \mathcal{O}_{K}$ at $\mathfrak{P}$ is nonzero. As $p$ is inert in $k$ the order of $\alpha \mathcal{O}_{K}$ at $\mathfrak{P}$ is even. Hence the order of $\sqrt{p} \alpha \mathcal{O}_{K}$ at $\mathfrak{P}$ is odd.

We can now prove Theorem 1.3. Clearly, we have

$$
\begin{aligned}
N\left(\sqrt{\mathbf{P}_{k}} k, X\right) & =1+\sum_{\substack{p \in \mathbf{P}_{k} \\
p \leq X^{2}}} N\left(\sqrt{p} k^{*}, X\right) \\
= & \sum_{\substack{p \in \mathbf{P}_{k} \\
p \leq X^{2}}} \frac{2 p^{d / 2}}{p^{d}+1} S_{k}(1) X^{2 d}+O\left(\frac{X^{2 d-1}}{p^{(d-1) / 2}}+X^{d} \log X+X^{d} \log p\right) \\
= & \sum_{\substack{p \in \mathbf{P}_{k} \\
p \leq X^{2}}} \frac{2 p^{d / 2}}{p^{d}+1} S_{k}(1) X^{2 d}+O\left(\sum_{\substack{p \in \mathbf{P}_{k} \\
p \leq X^{2}}} \frac{X^{2 d-1}}{p^{(d-1) / 2}}\right)+O\left(\sum_{\substack{p \in \mathbf{P}_{k} \\
p \leq X^{2}}} X^{d} \log X\right) .
\end{aligned}
$$

By the prime number theorem we have

$$
\sum_{\substack{p \in \mathbf{P}_{k} \\ p \leq X^{2}}} X^{d} \log X \ll X^{d+2} .
$$

A straightforward calculation yields

$$
\sum_{\substack{p \in \mathbf{P}_{k} \\ p \leq X^{2}}} \frac{X^{2 d-1}}{p^{(d-1) / 2}} \ll \begin{cases}X^{2 d-1} & \text { if } d \geq 4, \\ X^{5} \log \log X & \text { if } d=3, \\ X^{4} & \text { if } d=2 .\end{cases}
$$


To handle the first term let us start with the simpler case $d \geq 3$. Then we have

$$
\begin{aligned}
\sum_{\substack{p \in \mathbf{P}_{k} \\
p \leq X^{2}}} \frac{2 p^{d / 2}}{p^{d}+1} S_{k}(1) X^{2 d} & =\sum_{p \in \mathbf{P}_{k}} \frac{2 p^{d / 2}}{p^{d}+1} S_{k}(1) X^{2 d}+O\left(\sum_{\substack{p \in \mathbf{P}_{k} \\
p>X^{2}}} \frac{2 p^{d / 2}}{p^{d}+1} S_{k}(1) X^{2 d}\right) \\
& =\sum_{p \in \mathbf{P}_{k}} \frac{2 p^{d / 2}}{p^{d}+1} S_{k}(1) X^{2 d}+O\left(X^{2 d-1}\right) .
\end{aligned}
$$

This finishes the proof of Theorem 1.3 for $d \geq 3$.

Let us now assume $d=2$. It remains to show that

$$
\sum_{\substack{p \in \mathbf{P}_{k} \\ p \leq X^{2}}} \frac{2 p}{p^{2}+1}=\log \log X+O(1) .
$$

Clearly, we have

$$
\sum_{\substack{p \in \mathbf{P}_{k} \\ p \leq X^{2}}} \frac{2 p}{p^{2}+1}=\sum_{\substack{p \in \mathbf{P}_{k} \\ p \leq X^{2}}} \frac{2}{p}+O(1) .
$$

By an explicit version of Chebotarev's density theorem (see, e.g., [7]) we know that for $T \geq 3$ (using $\operatorname{Li}(T)=T / \log T+O\left(T /(\log T)^{2}\right)$ )

$$
\sum_{\substack{p \in \mathbf{P}_{k} \\ p \leq T}} 1=\frac{T}{2 \log T}+O\left(\frac{T}{(\log T)^{2}}\right) .
$$

Applying partial summation we get

$$
\sum_{\substack{p \in \mathbf{P}_{k} \\ p \leq X^{2}}} \frac{2}{p}=\sum_{m=2}^{X^{2}} \frac{1}{(m+1) \log m}+O(1)=\log \log X+O(1) .
$$

This completes the proof of Theorem 1.3 for $d=2$.

\section{A. Appendix}

We will now apply Theorem 6.1 to deduce the formula (1.8). We start by proving our claim that $\mathcal{N}^{\prime}$ is an adelic Lipschitz system whenever all the functions $N_{w}$ of $\mathcal{N}$ are norms. To this end we shall use the following simple observations. 
Let $f_{1}, f_{2}, f: \mathbb{R}^{q} \rightarrow \mathbb{R}$ and $F:[0,1]^{q-1} \rightarrow \mathbb{R}^{q}$ be functions that satisfy a Lipschitz condition with Lipschitz constant $L_{f_{1}}, L_{f_{2}}, L_{f}$ and $L_{F}$ respectively. Then we have:

1. $\left|f(F(\mathbf{t}))-f\left(F\left(\mathbf{t}^{\prime}\right)\right)\right| \leq L_{f} L_{F}\left|\mathbf{t}-\mathbf{t}^{\prime}\right|$ for all $\mathbf{t}, \mathbf{t}^{\prime} \in[0,1]^{q-1}$.

2. Suppose that $f(F(\mathbf{t})) \geq c>0$ for all $\mathbf{t} \in[0,1]^{q-1}$ and let $\alpha \leq 1$. Then $\left|f(F(\mathbf{t}))^{\alpha}-f\left(F\left(\mathbf{t}^{\prime}\right)\right)^{\alpha}\right| \leq|\alpha| c^{\alpha-1} L_{f} L_{F}\left|\mathbf{t}-\mathbf{t}^{\prime}\right|$ for all $\mathbf{t}, \mathbf{t}^{\prime} \in[0,1]^{q-1}$. (We use the convention that $0^{0}=1$.)

3. Suppose that $\left|f_{1}(F(\mathbf{t}))\right|,\left|f_{2}(F(\mathbf{t}))\right|,|f(F(\mathbf{t}))|,|F(\mathbf{t})| \leq C$ for all $\mathbf{t} \in[0,1]^{q-1}$. Then, for all $\mathbf{t}, \mathbf{t}^{\prime} \in[0,1]^{q-1}$,

(a) $\left|f_{1}(F(\mathbf{t})) f_{2}(F(\mathbf{t}))-f_{1}\left(F\left(\mathbf{t}^{\prime}\right)\right) f_{2}\left(F\left(\mathbf{t}^{\prime}\right)\right)\right| \leq C\left(L_{f_{1}}+L_{f_{2}}\right) L_{F}\left|\mathbf{t}-\mathbf{t}^{\prime}\right|$,

(b) $\left|f(F(\mathbf{t})) F(\mathbf{t})-f\left(F\left(\mathbf{t}^{\prime}\right)\right) F\left(\mathbf{t}^{\prime}\right)\right| \leq C L_{F}\left(L_{f}+1\right)\left|\mathbf{t}-\mathbf{t}^{\prime}\right|$.

Here 1. is obvious, 2. follows from the mean value theorem and 1., and 3. (a) and (b) are consequences of the identity $f g-f^{\prime} g^{\prime}=\left(f-f^{\prime}\right) g+f^{\prime}\left(g-g^{\prime}\right)$ and 1 . (note that the assumption $|F(\mathbf{t})| \leq C$ is needed only for (b)).

Lemma A.1. Let $\mathcal{N}$ be an adelic Lipschitz system (of dimension $n$ ) on $K$ and assume that for every Archimedean place $w$ of $K$ the function $N_{w}$ satisfies a Lipschitz condition. Then $\mathcal{N}^{\prime}=\mathcal{N}^{\prime}(\mathcal{N}, k)$ is an adelic Lipschitz system (of dimension $n$ ) on $k$.

Proof. The conditions (i), (ii) and (iv) in Definition 5.1 are obviously satisfied. It remains to prove (iii). Given an Archimedean place $v$ of $k$, let $\rho:[0,1]^{d_{v}(n+1)-1} \rightarrow$ $\mathbb{S}^{d_{v}(n+1)-1}$ be the (normalized) standard parameterization via polar coordinates of the $\left(d_{v}(n+1)-1\right)$-dimensional unit sphere in $k_{v}^{n+1}$. Then $\rho$ is Lipschitz. The subset of $k_{v}^{n+1}$ where $N_{v}(\mathbf{z})=1$ is parameterized by the function $\psi:[0,1]^{d_{v}(n+1)-1} \rightarrow$ $k_{v}^{n+1}$, defined by $\psi(\mathbf{t}):=1 / N_{v}(\rho(\mathbf{t})) \cdot \rho(\mathbf{t})$. Let us show that $\psi$ satisfies a Lipschitz condition.

For any Archimedean place $w$ of $K$ extending $v$, the function $N_{w}$ is continuous and nonzero on the compact set $\mathbb{S}^{d_{v}(n+1)-1}$, whence $1 \ll_{\mathcal{N}} N_{w}(\rho(\mathbf{t})) \ll_{\mathcal{N}} 1$ on $[0,1]^{d_{v}(n+1)-1}$. Thus, $N_{w}(\rho(\mathbf{t}))^{-\frac{d_{w}}{d_{v}[K: k]}}$ is bounded, and by 2 . satisfies a Lipschitz condition. Hence, by 3. (a) also $N_{v}(\rho(\mathbf{t}))^{-1}$ is Lipschitz. By 3. (b), we conclude that $\psi$ satisfies a Lipschitz condition.

Note that any norm $\|\cdot\|$ on $\mathbb{R}^{q}$ satisfies a Lipschitz condition. This follows from the reverse triangle inequality $|\|x\|-\|y\|| \leq\|x-y\|$ and the equivalence of all norms on $\mathbb{R}^{q}$. Thus, if all the functions $N_{w}$ are norms then Lemma A.1 applies and so $\mathcal{N}^{\prime}=\mathcal{N}^{\prime}(\mathcal{N}, k)$ is an adelic Lipschitz system (of dimension $n$ ) on $k$. More generally, let $B_{w}:=\left\{\mathbf{z} \in K_{w}^{n+1}: N_{w}(\mathbf{z}) \leq 1\right\}$ be the compact star-shaped body corresponding to $N_{w}$. Let $\operatorname{ker}\left(B_{w}\right)$ be the convex kernel of $B_{w}$, that is the set of all $\mathbf{z} \in B_{w}$ such that for all $\mathbf{z}^{\prime} \in B_{w}$ the line segment $\left[\mathbf{z}, \mathbf{z}^{\prime}\right]$ is contained in $B_{w}$. Then $\mathbf{0} \in \operatorname{ker}\left(B_{w}\right)$ and $B_{w}$ is convex if and only if $\operatorname{ker}\left(B_{w}\right)=B_{w}$. Moreover, [2, Lemma 1] tells us that $N_{w}$ is Lipschitz whenever $\mathbf{0}$ is in the interior of $\operatorname{ker}\left(B_{w}\right)$. 
Let us now show how the formula (1.8) follows from Theorem 6.1. We use the adelic Lipschitz system $\mathcal{N}$ (of dimension 2 ) on $K:=\mathbb{Q}(\sqrt{2}, \sqrt{3}, \sqrt{5}$ ) defined by

$$
N_{w}\left(z_{0}, z_{1}, z_{2}\right):=\max \left\{\left|z_{0}\right|_{w},\left|z_{1}\right|_{w},\left|z_{2}\right|_{w},\left|\frac{\sqrt{2} z_{1}+\sqrt{3} z_{2}}{\sqrt{5}}\right|_{w}\right\},
$$

for any place $w$ of $K$. Hence all the $N_{w}$ are norms so that, thanks to Lemma A.1, we can apply Theorem 6.1. With the notation from Section 6, we have $N_{L}(X)=$ $N_{\mathcal{N}}\left(\mathbb{P}^{2}(\mathbb{Q}), X\right)+O\left(X^{2}\right)$, as already mentioned in the introduction. Here the error term accounts for the projective points of the form $\left(0: \omega_{1}: \omega_{2}\right)$. With Theorem 6.1 , the only remaining task is to calculate $g_{\mathbb{Q}}^{\mathcal{N}}$.

Lemma A.2. We have

$$
g_{\mathbb{Q}}^{\mathcal{N}}=\frac{1}{31 \zeta(3)}\left(1+2 \cdot 5^{1 / 4}+4 \cdot 5^{-1 / 2}\right) .
$$

Proof. For some tedious computations in $K$, we use the computer algebra system Sage ${ }^{1}$. We use the same notation as in Section 6. Clearly, we can choose $\mathcal{R}=\{\mathbb{Z}\}$. For any $\boldsymbol{\omega}=\left(\omega_{0}, \omega_{1}, \omega_{2}\right) \in \mathbb{Q}^{3}$, we have

$$
i_{\mathcal{N}}(\boldsymbol{\omega})=\omega_{0} \mathcal{O}_{K}+\omega_{1} \mathcal{O}_{K}+\omega_{2} \mathcal{O}_{K}+\frac{\sqrt{2} \omega_{1}+\sqrt{3} \omega_{2}}{\sqrt{5}} \mathcal{O}_{K}
$$

If $\mathcal{O}_{\mathbb{Q}}(\boldsymbol{\omega})=\mathbb{Z}$ then $\omega_{0} \mathcal{O}_{K}+\omega_{1} \mathcal{O}_{K}+\omega_{2} \mathcal{O}_{K}=\mathcal{O}_{K}$, so $i_{\mathcal{N}}(\boldsymbol{\omega}) \supseteq \mathcal{O}_{K}$. On the other hand, we clearly have $i_{\mathcal{N}}(\omega) \subseteq(\sqrt{5})^{-1} O_{K}$. Thus, we can choose

$$
S_{\mathbb{Z}}:=\left\{(\sqrt{5})^{-1} \mathfrak{D}: \mathfrak{D} \mid \sqrt{5} \mathcal{O}_{K}\right\}
$$

Moreover, if $\omega \in \Lambda_{\mathbb{Z}}\left((\sqrt{5})^{-1} \mathfrak{D A}\right)$, for some nonzero ideal $\mathfrak{A}$ of $\mathcal{O}_{K}$, then $i_{\mathcal{N}}(\boldsymbol{\omega})=$ $(\sqrt{5})^{-1} \mathfrak{D}_{1}$, for some nonzero ideal $\mathfrak{D}_{1} \mid \sqrt{5} \mathcal{O}_{K}$. In particular, $\mathfrak{D} \mathfrak{A} \mid \mathfrak{D}_{1}$. This shows that $T_{\mathbb{Z},(\sqrt{5})^{-1} \mathfrak{D}}$ is contained in the finite set

$$
T:=\left\{\mathfrak{A}: \mathfrak{A} \mid \sqrt{5} \mathcal{O}_{K}\right\}
$$

With (6.6), we obtain

$$
g_{\mathbb{Q}}^{\mathcal{N}}=\sum_{\mathfrak{D} \mid \sqrt{5} \mathcal{O}_{K}} \mathfrak{N}_{K}\left((\sqrt{5})^{-1} \mathfrak{D}\right)^{3 / 8} \sum_{\mathfrak{A} \mid \sqrt{5} \mathcal{O}_{K}} \mu_{K}(\mathfrak{A}) \Sigma(\mathfrak{A D})
$$

${ }^{1}$ http://www.sagemath.org 
where

$$
\Sigma(\mathfrak{B}):=\sum_{n=1}^{\infty} \frac{\mu(n)}{\operatorname{det} \Lambda\left((\sqrt{5})^{-1} \mathfrak{B}, n \mathbb{Z}\right)} .
$$

Let us evaluate this sum for any ideal $\mathfrak{B}$ of $\mathcal{O}_{K}$ dividing $5 \mathcal{O}_{K}$. Elementary manipulations show that $\Lambda\left((\sqrt{5})^{-1} \mathfrak{B}, n \mathbb{Z}\right)$ is the sublattice of $\mathbb{Z}^{3}$ consisting of all

$$
\boldsymbol{\omega}=\left(\omega_{0}, \omega_{1}, \omega_{2}\right) \in\left(n \mathbb{Z} \cap(\sqrt{5})^{-1} \mathfrak{B}\right)^{3} \text { such that } \sqrt{2} \omega_{1}+\sqrt{3} \omega_{2} \in \mathfrak{B} .
$$

We have $5 \mathcal{O}_{K}=\mathfrak{P}_{1}^{2} \mathfrak{P}_{2}^{2}$, where

$$
\mathfrak{P}_{1}:=(5, \sqrt{15}-\sqrt{10}+\sqrt{6}-1), \mathfrak{P}_{2}:=(5, \sqrt{15}-\sqrt{10}+\sqrt{6}+1)
$$

are distinct prime ideals of $\mathcal{O}_{K}$ with inertia degrees equal to 2 .

For $\mathfrak{B}=\mathcal{O}_{K}$, the first condition in (A.2) amounts to $\omega \in(n \mathbb{Z})^{3}$. Then the second condition is always satisfied, and $\operatorname{det} \Lambda\left((\sqrt{5})^{-1} \mathcal{O}_{K}, n \mathbb{Z}\right)=n^{3}$. Therefore,

$$
\Sigma\left(\mathcal{O}_{K}\right)=\sum_{n=1}^{\infty} \frac{\mu(n)}{n^{3}}=\frac{1}{\zeta(3)} .
$$

If $\mathfrak{B}=\mathfrak{P}_{1}$, then the first condition in (A.2) is equivalent to $\omega \in(n \mathbb{Z})^{3}$. For the second condition, we find that $-(\sqrt{3})^{-1} \sqrt{2} \equiv 3 \bmod \mathfrak{P}_{1}$, so this condition is equivalent to $\omega_{2}=3 \omega_{1}+a$, for an $a \in \mathfrak{P}_{1} \cap n \mathbb{Z}=\operatorname{lcm}(5, n) \mathbb{Z}$. Therefore, $\Lambda\left((\sqrt{5})^{-1} \mathfrak{P}_{1}, n \mathbb{Z}\right)$ has the basis

$$
\{(n, 0,0),(0, n, 3 n),(0,0, \operatorname{lcm}(5, n))\}
$$

of determinant $n^{2} \operatorname{lcm}(5, n)$. A similar computation shows that $-(\sqrt{3})^{-1} \sqrt{2} \equiv 2$ $\bmod \mathfrak{P}_{2}$, so

$$
\{(n, 0,0),(0, n, 2 n),(0,0, \operatorname{lcm}(5, n))\}
$$

is a basis of $\Lambda\left((\sqrt{5})^{-1} \mathfrak{P}_{2}, n \mathbb{Z}\right)$ of the same determinant. Thus,

$$
\Sigma\left(\mathfrak{P}_{i}\right)=\sum_{n=1}^{\infty} \frac{\mu(n)}{n^{2} \operatorname{lcm}(5, n)}=\frac{1}{\zeta(3)} \frac{5^{2}-1}{5^{3}-1} .
$$

For $\mathfrak{B}=\mathfrak{P}_{1} \mathfrak{P}_{2}=\sqrt{5} \mathcal{O}_{K}$, the first condition in (A.2) is again equivalent to $\omega \in$ $(n \mathbb{Z})^{3}$. The second condition is equivalent to $\omega_{2} \equiv-(\sqrt{3})^{-1} \sqrt{2} \omega_{1} \bmod \mathfrak{P}_{1} \mathfrak{P}_{2}$. By the Chinese remainder theorem and what we have seen before, this is equivalent to

$$
\omega_{2} \equiv 2 \omega_{1} \quad \bmod 5 \quad \text { and } \quad \omega_{2} \equiv 3 \omega_{1} \quad \bmod 5,
$$


so $\omega_{1} \equiv \omega_{2} \equiv 0 \bmod 5$. Thus, $\Lambda\left((\sqrt{5})^{-1} \mathfrak{P}_{1} \mathfrak{P}_{2}, n \mathbb{Z}\right)=n \mathbb{Z} \times(\operatorname{lcm}(5, n) \mathbb{Z})^{2}$ has determinant $n \operatorname{lcm}(5, n)^{2}$. We obtain

$$
\Sigma\left(\mathfrak{P}_{1} \mathfrak{P}_{2}\right)=\sum_{n=1}^{\infty} \frac{\mu(n)}{n \operatorname{lcm}(5, n)^{2}}=\frac{1}{\zeta(3)} \frac{5-1}{5^{3}-1}
$$

In the other cases, that is $\mathfrak{P}_{1}^{2} \mid \mathfrak{B}$ or $\mathfrak{P}_{2}^{2} \mid \mathfrak{B}$, we have ${ }^{\mathfrak{d}}\left((\sqrt{5})^{-1} \mathfrak{B}\right)=5 \mathbb{Z}$, so the first condition in (A.2) is equivalent to $\omega \in(\operatorname{cm}(5, n) \mathbb{Z})^{3}$. In this case, the second condition is always satisfied, so we obtain $\operatorname{det} \Lambda\left((\sqrt{5})^{-1} \mathfrak{B}, n \mathbb{Z}\right)=\operatorname{lcm}(5, n)^{3}$ and

$$
\Sigma(\mathfrak{B})=\sum_{n=1}^{\infty} \frac{\mu(n)}{\operatorname{lcm}(5, n)^{3}}=0 .
$$

A simple computation shows that

$$
\begin{aligned}
& \mathfrak{N}_{K}\left((\sqrt{5})^{-1} \mathcal{O}_{K}\right)^{3 / 8}=5^{-3 / 2}, \quad \mathfrak{N}_{K}\left((\sqrt{5})^{-1} \mathfrak{P}_{i}\right)^{3 / 8}=5^{-3 / 4}, \\
& \mathfrak{N}_{K}\left(\mathcal{O}_{K}\right)^{3 / 8}=1 .
\end{aligned}
$$

To prove the lemma, just substitute this and (A.3) - (A.6) in (A.1).

\section{References}

[1] T. AngE, Le théorème de Schanuel dans les fibrés adéliques hermitiens, Manuscripta Math. 144 (2014), 565-608.

[2] G. A. BEER, Starshaped sets and the Hausdorff metric, Pacific J. Math. 61 (1975), 21-27.

[3] J. W. S. CAsSEls, "An Introduction to the Geometry of Numbers", Springer, 1997.

[4] J. H. EVERTSE, On equations in S-units and the Thue-Mahler equation, Invent. Math. 75 (1984), 561-584.

[5] X. GAO, “ On Northcott's Theorem”, Ph.D. Thesis, University of Colorado, 1995.

[6] T. LOHER and D. W. MASSER, Uniformly counting points of bounded height, Acta Arith. 111 (2004), 277-297.

[7] J. C. LAGARIAS and A. M. OdLyzKo, Effective versions of the Chebotarev density theorem, In: "Algebraic Number Fields: L-Functions and Galois Properties" (Proc. Sympos., Univ. Durham, Durham, 1975), Academic Press, London, 1977, 409-464.

[8] T. LoHER, "Counting Points of Bounded Height", Ph.D. Thesis, Universität Basel, 2001.

[9] D. W. MASSER and J. D. VAALER, Counting algebraic numbers with large height II, Trans. Amer. Math. Soc. 359 (2007), 427-445.

[10] D. MASSER and J. D. VAALER, Counting algebraic numbers with large height. I, In: "Diophantine Approximation", Vol. 16 of Dev. Math., Springer, 2008, 237-243.

[11] E. PEYRE. Hauteurs et mesures de Tamagawa sur les variétés de Fano, Duke Math. J. 79 (1995), 101-218.

[12] S. H. Schanuel, Heights in number fields, Bull. Soc. Math. France 107 (1979), 433-449. 
[13] W. M. SCHMIDT, "Diophantine Approximations and Diophantine Equations", Lecture Notes in Mathematics, Vol. 1467, Springer, 1991.

[14] W. M. SCHMIDT, Northcott's Theorem on heights I. A general estimate, Monatsh. Math. 115 (1993), 169-183.

[15] W. M. SCHMIDT, Northcott's Theorem on heights II. The quadratic case, Acta Arith. (1995), 343-375.

[16] C. L. STEWART, On divisors of Lucas and Lehmer numbers, Acta Math. 211 (2013), 291-314.

[17] M. WIDMER, Counting points of fixed degree and bounded height, Acta Arith. 140 (2009), 145-168.

[18] M. WIDMER, Counting points of fixed degree and bounded height on linear varieties, J. Number Theory 130 (2010), 1763-1784.

[19] M. WIDMER, Counting primitive points of bounded height, Trans. Amer. Math. Soc. 362 (2010), 4793-4829.

[20] M. WIDMER, Integral points of fixed degree and bounded height, Int. Math. Res. Notices, doi:10.1093/imrn/rnv268, first published online: September 25, 2015.

[21] K. YU, p-adic logarithmic forms and group varieties. III, Forum Math. 19 (2007), $187-280$.

[22] K. YU., p-adic logarithmic forms and a problem of Erdös, Acta Math. 211 (2013), 315-382.

Technische Universität Graz

Institut für Analysis und Zahlentheorie

Steyrergasse 30/II

8010 Graz, Austria

frei@math.tugraz.at

Department of Mathematics

Royal Holloway University of London Egham TW20 0EX, UK

Martin.Widmer@rhul.ac.uk 